\title{
Digitalisierung als kulturelle Praxis und ästhetisches Phänomen
}

Die Kultur-, Film- und Medienwissenschaft blickt nun auf mittlerweile über 3o Jahre Auseinandersetzung mit den sogenannten Computertechnologien und den einhergehenden (multi)medialen Phänomenen zurück. Nicht nur innerhalb einer kulturwissenschaftlichen Medienwissenschaft - auch in anderen Bereichen - ist das Feld extrem disparat, heterogen und damit recht unüberschaubar. Gerade deshalb ist es mit Blick auf das spezifische Interesse meiner Untersuchung an geschichtsbildmodellierenden und erinnerungsbildenden Prozessen digitalisierter Filme zentral, einige Positionen zu bestimmten medialen und soziokulturellen Entwicklungen und Phänomenen historisch und ideengeschichtlich einzuordnen. Das Kapitel stellt kumulativ ausgewählte Ansätze und Perspektiven unter Berücksichtigung des jeweiligen medienhistorischen Kontextes dar, die für die Gesamtargumentation von Relevanz sind. Es geht weniger um die Entwicklung einer zentralen These als vielmehr um eine Auffächerung der Vielfalt der Betrachtungsmöglichkeiten des Phänomens digital, die von bestimmten technischen Grundbedingungen digitaler Bilder verstärkt werden. Grundlegendes Ziel ist es, den Komplex digital als kulturelle Praxis sowie als vom Kontext abhängiges ästhetisches Phänomen für die anschließenden Kapitel zu würdigen und zudem wiederkehrende Diskurstopoi zu extrapolieren. Auch gilt es hierbei, entsprechend der Terminologie des kulturgeschichtlichen Ansatzes von H. Böhme (2000) auf die Imagologie der digitalen Domäne, das spezifisch Technoimaginäre, hinzuweisen.

Dieses $\rightarrow$ Kap. 2 gliedert sich in zwei Teile. Der erste Teil beschäftigt sich mit Begriffen und Konzepten. Hier werden die technischen Grundlagen digitaler Bilder und ihrer ästhetischen Erscheinungsformen erläutert. Dies schließt, wie die Argumentation zeigen wird, für den vorliegenden Gegenstandsbereich fundamental an die Verhältnisbildung analog/digital an; zugleich bedeutet dies eine Re-Modellierung von Vorstellungen des Mediums Film im Horizont von Digitalisierungsprozessen, was anschließend erläutert und diskutiert wird. In der Folge wird der Begriff digital aus kulturwissenschaftlicher Perspektive im Horizont medienhistoriografischer Konzepte präzisiert. Dies führt zu methodologischen Überlegungen, die nicht abzulösen sind von fachpolitischen und disziplinären Debatten, die sich in der Perspektivierung des Verhältnisses von 
Technologieentwicklung, Medienbegriff, kultureller Rahmung, Vorstellungen von historischer Dynamik und ästhetischen Strukturen manifestieren. In diesem Abschnitt wird zum einen der kulturkritische Ansatz der Studie geschärft, zum anderen der Begriff der Transition als Konzept von Geschichtlichkeit angesichts von medialen Phänomenen hergeleitet.

Der zweite Teil dieses Kapitels (Digitalisierung, Gesellschaft, Ästhetik und Geschichte) markiert vor allem den Übergang zur Beschäftigung mit Positionen, die noch stärker die soziokulturelle Umgebung digitaler Technologien untersuchen und diese auf ihre impliziten Ideologien hin analysieren. Hier gilt das besondere Augenmerk den Auswirkungen konkreter Phänomene auf Konzeptionen von Ästhetik, Geschichtlichkeit und Geschichte. Dies wird an einem historischen, vordigitalen Fallbeispiel der marktorientierten ästhetischen Anpassung von Filmwerken angesichts neuer Medienumgebungen veranschaulicht: konkret am Beispiel der in den USA der 1980er Jahre geführten Colorization-Debatte, die durch die elektronische/maschinelle Nachkolorierung von Filmklassikern für das Fernsehen ausgelöst wurde. Insbesondere mit diesem historischen Fallbeispiel werden Praktiken der Medienindustrie und deren Konsequenzen für populäre Gedächtnisbildung sichtbar, die noch vor der eigentlichen sogenannten digitalen Revolution grundlegende Fragen aufwarfen. Zudem ermöglicht dieses Beispiel eine Präzisierung des Gegenstandbereiches dieser Studie mit Blick auf den Begriff von Populärkultur und die dort stattfindenden Geschichtsbildmodellierungen. Auch wird hier deutlich, welch dynamisierende Funktion die Digitalisierung von Filmen aus dem Archiv - in einem weiteren Sinne als das Verfahren der Colorization als (erinnerungs)kulturelle Praxis für unser Geschichtserleben einnimmt. Zur Veranschaulichung der Thesen schließt das Kapitel mit einem weiteren, diesmal jüngeren Beispiel aus der deutschen Fernsehpraxis, das sich dezidiert nicht mehr im Bereich von historischen Spielfilmen, sondern im Bereich der NonFiction bewegt und damit den Anspruch erhebt, Geschichte wiederzugeben aktualisiert und ästhetisch angepasst mit digitalen Technologien.

\section{Begriffe und Konzepte}

\subsection{Die Ästhetik digitaler Bilder als Interpretationsvorgang}

In der Grundlagenliteratur zur digitalen Bild- und Videotechnik aus den 199oer Jahren wie etwa Charles Poyntons Introduction to Digital Video (1996) werden vor allem vier Begriffe als Basisprinzipien genannt: Quantisierung, Auflösung, 
Kompressionsalgorithmen, Abtastung. Hier wie auch in spezialisierten Handbüchern - etwa zu dem weiteren zentralen Thema der Verarbeitung von Farbinformationen ${ }^{1}$ - wird deutlich, dass eines der Hauptaugenmerke in der Beschäftigung mit digitalen Bildern die polymorphe Eigenschaft der Informationen ist, wobei digital bedeutet, dass die Informationen im Grundprinzip in einer binären Struktur - 1 und o als diskrete, in sich abgeschlossene Werte - vorliegen.

Die jeweilige Verarbeitung, das heißt Interpretation der Informationen in unterschiedlichen Umgebungen sowie die Kommunikation von Daten, stellt das Zentrum der Diskussion dar und wird deshalb in spezialisierten Studien in den Blick genommen. ${ }^{2}$ Denn dies ist wesentlich für die Vorstellung, dass digitale Informationen immer eines Interpretationsvorgangs bedürfen: Digitale Informationen, als numerische Werte definiert, besitzen keine inhärente visuelle Bedeutung (Stone 2003).

Allerdings wird die Abhängigkeit der Interpretation der digitalen Daten von ihrem Kontext in den entsprechenden Einführungen im Rahmen dieses technisch geführten Diskurses nicht auf pragmatische, soziokulturelle Umgebungen ausgeweitet - wie etwa auch auf eine mögliche Historizität von ästhetischer Wahrnehmung und von Bildkonzepten. Insgesamt herrscht in dieser Form der Einführungsliteratur das Interesse an den technischen Problemen der digitalen Domäne und Bildgebungsverfahren vor, eine soziokulturelle und ästhetische Kontextualisierung wird hier kaum unternommen.

Eine der wenigen Ausnahmen ist die Arbeit von Barbara Flückiger seit den 2oooer Jahren, da sie in ihrem "technobolen Ansatz" (Flückiger 2008: 16) technische Aspekte mit den ästhetischen Konsequenzen verbindet, die sie unter anderem als historisch geprägt und gewachsen versteht. Insofern ist es schwer - wie Flückiger ausführlich darstellt (2008: $3_{1} \mathrm{ff}$.) -, grundlegende Eigenschaften von digitalen Bildern überhaupt zu bestimmen. ${ }^{3}$ Flückiger

1 Handbücher wie das der GretagMacbeth Group (1999) bieten anschauliche Basiserklärungen zur Farbmischung, Farbtemperatur sowie zur Kalibrierung unterschiedlicher Ein- und Ausgabesysteme (etwa Scanner, Monitor).

2 Diese Elemente spielen in der Digitalisierung beziehungsweise in den Scanprozessen von Filmen eine zentrale Rolle und bestimmen das spätere Digitalisat (Wittmann 1999; zur Lichtbestimmung/zum Grading vgl. Hullfish 2008; Flückiger 2012; Flückiger/Heller/Op den Kamp et al. 2016).

3 Simon Rothöhler hat zuletzt den Diskurs um die grundlegenden Eigenschaften digitaler Bilder in eine bildtheoretisch logistische Perspektive gerückt, indem er digitale Bilder in ihren besonderen mobilen Eigenschaften fasst, die Transportkalkülen gehorchen. Er folgt damit einem Ansatz, der sich von Flückigers „technobolem“ Ansatz vor allem in der theoretischen Reichweite und dem Bezug zur konkreten Populärkultur unterscheidet (vgl. Rothöhler 2018). Eine wichtige Referenz stellt für Rothöhler etwa Claus Pias (2003) dar. 
nennt deshalb nur einige wenige Parameter, die allen digitalen Bildern gemein seien: Digitale Bilder bestünden aus diskreten Feldern, den Pixeln, deren Eigenschaften durch einen binär codierten mathematischen Wert definiert seien. Diese Pixel seien gitterförmig - horizontal und vertikal - angeordnet (Flückiger 2008: 31).

Um den Gegensatz zur ,analogen' Domäne, das heißt vor allem auch zu fotochemischen bildgebenden Prinzipien hervorzuheben, die im Aufnahmeverfahren tatsächlich fotochemisch und physikalisch mit der außermedialen Realität interagieren, zitiert Flückiger Mitchell (1992). Dieser beschreibt anschaulich die Differenz der Art und Weise, wie eingehende Signale als kontinuierlicher physikalischer Fluss in Informationen und dann über den Kontakt mit einem fotochemischen Material in ein Bild umgewandelt werden: Analoge Signale könnten unendlich viele Werte annehmen, so entsteht ein kontinuierlicher Fluss in den Werten.

Wird ein Signal digitalisiert, bedeutet dies eine mathematische Zerlegung des Signalflusses in eine diskrete, binäre Struktur, das heißt, jedem Wert wird ein einzelner, in sich abgeschlossener, von den anderen Werten abgegrenzter Wert zugeordnet. Mitchell (1992) illustriert den Unterschied mit dem differenziellen Bild einer Rampe und einer Treppe: „Rolling down a ramp is a continuous motion, but walking down stairs is a sequence of discrete steps - so you can count the number of steps, but not the number of levels on a ramp" (Mitchell 1992: 4). Diese Stufen werden - dem abstrahierenden Prozess als mathematische Vermessung folgend - durch Bits codiert, die wiederum in Bytes organisiert sind. Ein Bit (binary digit) ist die kleinste Informationseinheit in diesem System, bestehend aus o oder 1. Dieser Prozess der Zerlegung in diskrete Werte ist der Prozess der Quantisierung, in dem die Werte mathematisch in einem Intervall eindeutig zugeordnet werden. Die Zuordnung funktioniert nur im Rahmen eines bestimmten, vordefinierten Bereichs. Mit anderen Worten, die Quantisierung erfolgt nach Regeln, die festlegen, was im darstellbaren Bereich des entstehenden Bildes liegt und was nicht. Was außerhalb des codierbaren Bereichs liegt, wird ignoriert oder den maximalen äußeren Grenzen zugeordnet (Flückiger 2008: 35$) \cdot{ }^{4}$

So zeigt Flückiger nicht nur die technische Bedeutung dieser Prozesse als Grundeigenschaften auf, sondern macht auch auf die kulturellen und memopolitischen Konsequenzen aufmerksam:

4 Als etwas verkürztes Anschauungsbeispiel: Ist etwa der als schwärzester Punkt eines Farbraumes definierte erreicht, kann er nicht überschritten werden. Vgl. hierzu das Phänomen des Clippings bei Flückiger (2008: 35). 
Die binäre Kodierung ist nicht nur das entscheidende Grundcharakteristikum aller digitaler Daten und damit selbstredend aller digitalen Bilder, sondern auch der Kern- und Angelpunkt ihrer ästhetischen und kulturellen Wirkung. Aus dieser Darstellung geht hervor, dass Quantisierung ursprünglich eine Vereinfachung darstellt, denn die diskrete Kodierung in Stufen führt zum Verlust aller Nuancen dazwischen (Flückiger 2008: 35).

So bieten digitale Bilder gleich auf zwei Ebenen Anlass zur Interpretation:Zum einen auf dem Niveau der ursprünglichen ,analogen“ Signalverarbeitung, da sie grundsätzlich eine abstrahierende Zerlegung in mathematische Prinzipien bedeuten. Mit den Worten Flückigers: „Wenn analoge Daten digitalisiert werden, sei das durch digitale Aufnahme oder durch Scannen, findet eine Übertragung nach bestimmten Regeln statt“ (Flückiger 2008: 35$) \cdot{ }^{5}$ Diese Regeln sind zunächst mathematisch und durch die Aufnahmeapparatur bestimmt. Wie aber vor allem Flückiger/Heller/Op den Kamp et al. 2016 nachgewiesen haben, können sich diese Vorgänge ebenfalls in einem viel größeren soziokulturellen Netzwerk bewegen, welches mit den technischen Parametern interagiert und jeweils die digitalen ,Erfassungsregeln' bestimmen kann.

Zum anderen tragen die Daten keine visuelle Bedeutung in sich, sie müssen erst als Bilder in ihrer Erscheinungsform decodiert werden. Auch dies wird von verschiedenen Faktoren bestimmt, die über den zunächst erst einmal augenscheinlich computerisierten Vorgang einer Decodierung hinausgehen. So werden etwa in der Festlegung einer Erscheinungsform als Ziel - wie das Bild auszusehen hat, das man aus den Daten extrapoliert - bereits kulturelle und ästhetische Setzungen vorgenommen, was man im gegebenen Kontext unter

5 In Hinblick auf den konkreten Transitionsprozess physikalischer Phänomene in die digitale Domäne weist Flückiger auf die Bedeutung einer Doppelperspektive hin: Nicht nur muss man die digitale Domäne in ihren Funktionsprinzipien verstehen, man muss auch die ästhetischen und wirkungstheoretischen Prämissen des Ausgangsmaterials kennen. Danach gilt es, die Bedingungen (,Regeln') der Transition zu modellieren, wobei sich die ,Regelbildung im technischen Ablauf noch einmal aufgrund der Inputdaten wiederholt. Es muss eine Analyse stattfinden, in deren Verlauf das physikalische Phänomen oder die analogen Bilddaten nach vorgeschriebener Routine in die binären Codes transformiert werden. Dazu entnimmt man den zu verarbeitenden Daten in zeitlichen und örtlichen Intervallen Stichproben. Diesen Vorgang bezeichnet man als Abtastung (sampling). Entweder tastet man mit einer Stichprobe (sample) pro Pixel ab oder mit mehreren Stichproben (oversampling) oder mit weniger als einem Sample (subsampling). Filtern ( filtering) legt die örtliche und zeitliche Verteilung des Abtastens fest und definiert, welche Ausschnitte aus dem Datenfluss welchen Stichproben zugeführt werden, um Zufallsartefakte als Bildfehler zu vermeiden. Die analogen Ausgangsdaten des vorliegenden analogen (Film-)Bildes müssen also erst analysiert werden, bevor die Quantisierungsregeln greifen können, nach denen man sie in die binären Codes überträgt. Die Kombination von Abtasten und Filtern stellt einen grundlegenden Prozess der digitalen Bilderzeugung dar (Flückiger 2008: 35-36). 
dem beziehungsweise noch grundsätzlicher einem Bild versteht - unter Umständen mit Bezug auf eine Referenz aus der analogen (Medien-)Welt (dazu später mehr). Hier werden vielfältige Mechanismen nachhaltig wirksam, die ich in ihrer kulturgeschichtlich und damit memopolitisch formierenden Dimension untersuche.

Die Notwendigkeit, Daten in ihrer Manifestation als Bilder in ihrer ästhetischen Erscheinungsweise einem technischen wie kultur- sowie kunstpolitisch geprägten Interpretationsvorgang zu unterwerfen, damit sie sichtbar und erfahrbar werden, zeitigt Konsequenzen für die Beschäftigung mit dem Phänomen digitaler Bilder in geisteswissenschaftlicher, insbesondere medienanalytischer und -ästhetischer Perspektive. Flückiger kritisiert hier, dass in der medien- und kulturwissenschaftlichen Literatur vor allem Übergeneralisierungen vorgenommen werden, in denen zu einfache Kausalzusammenhänge zwischen dem Darstellungsprinzip digital und produktionstechnischen Praktiken sowie ästhetischen Erscheinungsweisen vorausgesetzt werden. So stellt die Frage nach dem Status des digitalen Bildes dem ,digitalen Bild an sich - einen Schwerpunkt der Debatte insbesondere in der deutschsprachigen Forschung dar. Ein solcher Status wurde vor allem über die Konstruktion von Gegensatzpaaren konstruiert (Flückiger 2008: 13 ff.). ${ }^{6}$

Vor diesem Hintergrund konstatiert Flückiger 2008 mit Blick auf die Forschungsliteratur:

6 Diese methodologische Problematik wird deutlich, betrachtet man Publikationen aus den 2oooer und 2010er Jahren. Es gibt zahlreiche Veröffentlichungen in Sammelbänden, die informative Einblicke in Einzelphänomene digitaler Bilder aus unterschiedlichsten Perspektiven liefern (Segeberg 2012a; Schröter/Stiglegger 2011; Slansky 2004). Oft geschieht dies unter unterschiedlichsten Vorzeichen und Perspektiven, was einen allgemeinen Überblick schwer macht. Ein Problem der fehlenden Übersicht liegt vor allem darin begründet, dass die jeweilige digitale Technik ganz unterschiedlich begriffen werden kann. Je nach Ausgangsdefinition formiert sich die Idee der technischen Bedingungen, die unter dem Begriff digital gefasst werden. Diese werden in unterschiedliche Kausalitätsketten zwischen technologischer Bedingung und der entstehenden Bildästhetik eingeordnet. Dies kann von der Auseinandersetzung mit den Kameras beziehungsweise den hier verwendeten Sensoren und Chips als Erfassungs- und Transformationssysteme (Hahn 2005) bis hin zur Beschäftigung mit der Mobilität und Handlichkeit der Kameras reichen - auch mit Anbindung an einen Überwachungsdiskurs und soziale Medien (Rothöhler 2013; Kammerer 2008; Kulle 2013). HD als Schlagwort wird wiederholt mit der Bestimmung digitaler Ästhetik in Zusammenhang gebracht (Rothöhler 2013; Ritzer 2011); Interaktivität, Immersion (u. a. Neitzel/Nohr 20o6) und Echtzeit (Otto/Haupts 2012) als Bestimmung einer sogenannten ,digitalen Kultur'/,Digitalkultur' stellen weitere wiederkehrende Topoi dar. Aspekte der Indexikalität beziehungsweise simulatorische und mimetische Eigenschaften von digitalen Daten in Hinblick auf ,ältere Medienphänomene wie analoge Filmbilder schließen an die Abbildungsproblematik an. Die Abbildungsproblematik ist wohl eine der zentralen (historischen) Topoi in der Auseinandersetzung um digitale Bilder (Doll 2012). 
Viele Medientheoretiker befassen sich sozusagen mit dem ,digitalen Bild an sich', einer gleichsam überhöhten Simplifizierung des riesigen Gebiets von Anwendungsfeldern des digitalen Bildes, das [...], wo immer es erscheint, ebenso sehr von seinem kulturellen Kontext geprägt sein wird, wie es sich wiederum auf diesen Kontext auswirkt (Flückiger 2008: 19).

So bringt es Flückiger 2012 auf die Formel: „Es gibt nicht das digitale Bild, sondern nur eine Vielfalt von ihm“ ("there is no such thing as the digital image, only a variety thereof") (Flückiger 2012: 10, Hervorh. FH). ${ }^{7}$

Maulko teilt Flückigers Abkehr von einem essenzialistischen Konzept von digital, wenn er schreibt:

Digitalästhetik ist zu wesentlichen Teilen das, was wir speziell in kulturellen, wahrnehmungsästhetischen und visuellen Diskursen daraus machen, was wir dafür halten und was wir in verschiedenen Phasen der Entwicklung den Visualisierungen zuschreiben (Maulko 2012: 53).

Was Flückiger als „Übergeneralisierungen“ beschreibt und worauf auch Maulko mit Blick auf „digitale Ästhetik“ aufmerksam macht, kann als zentrales Problem der Forschung im Zeichen der Medientransition analog/ digital ausgemacht werden: Oft wird in Studien lediglich ein Aspekt digitaler Bilder herausgegriffen und fokussiert. Dieser ausgewählte Aspekt wird als bestimmendes Charakteristikum begriffen und auf ästhetische Merkmale der konkreten visuellen Manifestation bezogen, was dann unter Umständen zur Behauptung einer kausalen Beziehung zwischen ausgewähltem Aspekt und digitaler Bildtechnologie führt. 8

Ich vertrete einen anderen Ansatz: Insbesondere bei mediengeschichtlichen oder -ästhetischen Fragestellungen ist vor allem die fehlende Stabilität und Identität von digitalen Bildern immer mitzudenken. Dass eine konkrete (audio)visuelle Manifestation bereits die sinnliche Form eines kulturpolitisch wirksamen Diskurses im Modus des Ästhetischen darstellt, ist als eine zentrale Herausforderung dieses Problemfeldes zu sehen.

Die vielschichtigen Interpretationsvorgänge, die bestimmen, wie ein digitales Bild zu seiner Erscheinung kommt, sind nachhaltig kontextuell und

7 Flückiger differenziert weiter aus: „There is a big difference whether a digital image is captured with a digital camera or whether it was generated by means of computer software. In addition, there are processes that translate analog images into the digital domain, either to render them with software for compositing, along with other post-production tools such as editing or color grading, and/or to display them on electronic devices" (Flückiger 2012: 10).

8 Eine Publikation, die sich dieser Problematik bewusst ist, ihr aber trotzdem nicht ganz entgeht, ist Rothöhlers Buch (2013) zu digitaler Filmästhetik im Horizont von High Definition. 
situativ gebunden. Dies gilt auf der Ebene des Scannens, der Datenverarbeitung wie auch zusätzlich für die Speicher- und Wiedergabegeräte, das heißt für die jeweilige Medienumgebung, in deren Rahmen die Bilder sich manifestieren. Gerade die Wahl und der konkrete Gebrauch des Rezeptionsmediums beinhaltet einen weiteren, nicht zu vernachlässigenden Interpretationsvorgang. Es enwickelt sich hier eine komplexe Wechselwirkung: Die Bilder und ihre Erscheinungsformen stehen im Zusammenhang mit (historisch gewachsenen) Vorstellungen etwa von Ästhetik und ,Schönheit'; gleichzeitig werden diese Vorstellungen an die Bedingungen gegenwärtiger Medienumgebungen angepasst. Als Teil der aktuellen Medienkultur nehmen die Bilder mit ihren Erscheinungsweisen dann wieder Einfluss auf die Geschmacksbildung und Erwartung an (historische) Filme und deren Aussehen. Damit findet eine ständige, wechselseitig wirkende Aushandlung der Bedingungen der Erscheinungsweisen digitaler Bilder statt - zwischen vergangenen, konditionierend wirkenden medialen Praktiken und gegenwärtigen Manifestationsformen.

\subsection{Die Relation analog/digital als Bedingung der Erscheinungsform digitaler Bilder}

Bei der kulturellen Prägung hinsichtlich des Aussehens medialer, das heißt hier technisch reproduzierter Bilder, nimmt der Begriff analog als historische Referenz eine zentrale Rolle ein. Insbesondere vor dem Hintergrund, dass digitalen Bildern eine visuelle Identität, wie sie ontologisch etwa an analogen Bildern medienhistorisch festgemacht wurde, fehlt, formuliert Schröter die Notwendigkeit der ,Bändigung dieser Unbestimmtheit durch die historisch gewachsene und konditionierend wirkende Medientechnik des Analogen:

\footnotetext{
[M]an [könnte] mit einem durch die Kittler-Brille gelesenen Luhmann das Digitale als Medium begreifen, welches durch analoge Formen strikter gekoppelt wird, ja werden muss, da es selbst leer und offen, mithin absolut unbestimmt ist. Dann kämen nicht die digitalen nach den analogen Medien, sondern vielmehr würden die analogen Medien durch ihr digitales Sampling, ihre digitale Simulation zugleich beendet wie fortgesetzt [...]: Indem die digitalen Techniken auf die bislang von den analogen Medien gehaltenen, ,medialen Plätze' einrücken [...], werden sie erst Medien. Die digitalen Medien wären sozusagen nur ein neuer Aggregatzustand ihrer analogen Vorläufer (Schröter 2004a: 24).
}

Mit dem Rekurs auf die Möglichkeiten, mittels digitaler Informationen analoge Verfahren simulieren zu können, betont Schröter die Herausforderung, die digitale Daten als ästhetische Ausdrucksform für die Konzeption von 
mediengeschichtlicher Dynamik darstellen. Nicht nur ist der Übergang von analogen zu digitalen Medientechniken kaum als Bruch anzusehen, vielmehr, so Schröters und Flückigers Argument, werden Erfahrungen aus der analogen Mediengeschichte benötigt, um digitale Bilder als solche sicht- und lesbar zu machen. Dies wird in einem weiteren, später folgenden Unterkapitel als medienhistoriografische Herausforderung mit dem Begriff der Transition präzisiert werden. ${ }^{9}$

Schröter resümiert die Diskurslinien, die sich um das medientheoretisch zentrale Differenzverhältnis analog/digital ranken und in der Unbestimmtheit von digital begründet liegen. Seine Befunde lassen sich in ihrer ideologischen Dimension in Hinblick auf die Vermittlung von temporalen Relationen durchaus bis heute erkennen:

Mit der [seit Beginn der 199oer Jahre, FH] überall ausgerufenen, digitalen Revolution' kamen die ,Neuen Medien' - und mit diesen die angeblich für immer prosperierende ,New Economy', die ,Hyperrealität' oder doch mindestens das ,Ende des fotografischen Zeitalters'. ,Digital' ist mittlerweile ein buzzword geworden, das fast jedem Phänomen zugeordnet werden kann. Es konnotiert auf diffuse Weise ,neu', ,fortschrittlich` und ,computer-technisch'. Demgegenüber scheint alles Analoge auf unbestimmte Weise hoffnungslos veraltet (Schröter 2004a: 7).

Gerade durch seine Unbestimmtheit benötigt digital - nach Schröter - in der mediengeschichtlichen Narration die Differenz zu analog. Die Macht der Unterscheidung liegt vor allem in der ideologisch interessegeleiteten Konstitution einer hierarchischen Opposition. In dieser Verhältnissetzung ist das Analoge ${ }^{10}$ als das Ursprüngliche, Natürliche, Reale oder kausal mit dem Realen Verbundene, als das Amorphe konnotiert. Das Digitale hingegen steht in dieser Perspektive für das Omnipotente, Universelle, ja Kulturelle (Schröter 2004a: 14).

Diese diskursanalytisch ausgerichteten Befunde Schröters sind insofern wichtig, als sie die interessegeleitete, vom Kontext abhängige ideologische Aufladung der Images zweier Medientechnologien aufzeigen, die sich in Bildgebungsverfahren als Praktiken - besonders im Kontext der Debatte um Filmbilder - manifestieren. Begründet in der Unbestimmtheit der einen

9 Schröter diskutiert den historiografisch modellierenden Begriffsgebrauch wie folgt: „Medienumbrüche sind keine absoluten Einschnitte und Risse, sondern Umordnungen komplexer Konstellationen. Deswegen heißen sie auch Umbrüche im Gegensatz zu Brüchen" (Schröter 2004a: 29).

10 Der substantivierende Begriffsgebrauch wird hier punktuell von der Quelle übernommen, da es hier um die homogenisierend wirkenden Images von Medientechnologien geht. 
(digital), unterliegt die andere (analog) einer Homogenisierung im Sinne einer Festschreibung auf ein differenzielles Prinzip, das in diesem Narrativ die Konnotation eines fass- und fixierbaren Ursprungs vermittelt. ${ }^{11}$ Hier wird diskursiv die Voraussetzung dafür geschaffen, dass etwa analoge Filmgeschichte als etwas natürlich Gegebenes konstruiert werden kann, welches dann technizistisch (= digital) im medientechnologischen Fortschrittsnarrativ kulturell fassbar, aktualisiert und zugänglich gemacht wird. ${ }^{12}$

\subsection{Zwischenfazit: Images, Mythen und Versprechen neuer Medien}

Mit Blick auf die technologische Entwicklung lässt sich ein historisches Kontinuum vom mechanischen über das elektronische bis hin zum digitalen Zeitalter nachzeichnen. Damit wird ein Trend sichtbar, der weg von dem konkret Haptischen hin zu Modi der Abstraktion führt (Flückiger 2008: 43). Damit einher geht auch die Auflösung der Annahme einer unverbrüchlichen Verquickung von Material und Wahrnehmungsgegenstand hin zu einer eher losen Verbindung, was Le Grice (1998) als akzidentell bezeichnet. Unter diesen Vorzeichen, so meine Konklusion, rücken die Umstände und Kontexte von Wahrnehmungsgegenständen und -phänomenen als sinn- und bedeutungskonstituierende Faktoren in den Fokus.

Im vorangegangenen Abschnitt wurde in mehrfacher Hinsicht hervorgehoben, wie digitale Informationen an sich polymorph sind. Das heißt je nach Aufnahme-, Speicher-, Wiedergabe- und Ausgabesystem sind die Erscheinungsformen von digitalen Bildern wandelbar. Damit stellt sich auf

11 Dies zeitigt auch Konsequenzen für das Verständnis der mediengeschichtlichen Dynamik im Horizont eines radikalen Umbruchs: „Sicher hat sich [...] die emphatische Reduktion der komplexen medialen Transformationen und Verschiebungen auf eine ,digitale Revolution' überlebt. Das ist schon daher naheliegend, weil die Behauptung eines reinen Übergangs vom Analogen zum Digitalen ausblenden muss, dass analog und digital immer nur differenziell aufeinander bezogen Sinn machen" (Schröter 2004a: 29).

12 Schröter zeigt in seiner Diskursanalyse, wie nicht nur der Bildästhetik, sondern auch digitalen Speicher- und Wiedergabemedien allgemein ein ,intensiveres' (Abbildungs-) Verhältnis zur Wirklichkeit zugeschrieben wurde - allerdings in einer sich durchaus paradox entwickelnden Spirale der Konnotationen: „Mit der CD wurde die Analog/ Digital-Differenz immer bekannter, ja ganz konkret fassbar, und mit ihr die genealogisch angelegten Konnotationen: Euphorisch wie kulturkritisch etablierte sich die unterkomplexe Dichotomie analog = real, aber auch mit den Trübungen der Welt behaftet vs. digital = hyperreal, also realer als real, aber potenziell auch irreal“ (Schröter 2004a: 16). 
verschiedenen Ebenen die Frage, wie man den Referenzrahmen setzt, um die Ästhetik der Bilder zu definieren und zu qualifizieren. ${ }^{13}$

Wie bereits mit Rekurs auf Schröter angedeutet wurde, nun aber noch medienspezifisch erweitert werden muss, bedingen digitale Bilder als Filmbilder ein besonderes Verhältnis zur Medien- und Filmgeschichte, da Film als historisch älteres Medium zu einem heterogenen Referenzrahmen für Ästhetik, Narration und dispositivische Strukturen werden kann. Im diskursanalytischen Ansatz Schröters wurde deutlich, wie über die diffusen Images von analog wie digital historiografisch wirksame Setzungen vorgenommen werden; dies vor allem mit Blick auf temporale Verhältnisse, insbesondere auf die Konstitution von zeitlicher Distanz.

Hierbei entscheidend ist die hierarchische Anordnung von analog/digital, die ihren Ursprung in den technischen Möglichkeiten der digitalen Domäne der Simulation ${ }^{14}$ und Emulation ${ }^{15}$ hat, sich aber dann ideologisch in ein Fortschritts- und Überlegenheitsnarrativ einschreibt - mit praktischen kultur- und memopolitischen Folgen. ${ }^{16}$

Dies spiegelt sich auch in dem Begriffsgebrauch um die Jahrtausendwende, in dem versucht wird, zum einen den technologischen Images zu entkommen, zugleich aber affirmativ eine zeitlich-hierarchische Ordnung unter der Maßgabe des ,Neuen' vorzunehmen: Der Begriff ,Neue Medien' wurde vor allem von Lev Manovich stark gemacht. Distelmeyer kommentiert retrospektiv den Begriffsgebrauch zur Hochzeit der grundsätzlichen publizistischen Auseinandersetzungen mit der digitalen Domäne wie folgt:

13 Der Referenzrahmen kann unter diesen Vorzeichen nicht allein in Hinblick auf die Bildästhetik gesehen werden, sondern muss gerade in digitalen Dispositiven als Wiedergabeund Zugangssysteme (etwa DVD oder Blu-Ray-Disc) auch in der jeweiligen zeiträumlichen verhältnissetzenden Anordnung sowie in den diskursiven oder paratextuellen Kontextualisierungen verortet werden.

14 Ich folge hier Flückigers (2008: 517) doppeltem Gebrauch von ,Simulation': Zum einen wird der Begriff im Kontext von Computersimulation als regelbasiertes Modell zur Beschreibung und Entwicklung und/oder zum Verhalten von komplexen Systemen verstanden; zum anderen aber auch im soziologisch-philosophischen Verständnis etwa bei Baudrillard (1978) als Vorspiegelung oder Vortäuschung eines Sachverhalts oder Vorwands. Vgl. auf die Simulation analoger Filme im Rahmen digitaler Restaurierungen bezogen Fossati (2009: 140-145).

15 Unter Emulation wird hier die mögliche Nachahmung verschiedener Eigenschaften eines Geräts oder einer Software mithilfe anderer Programme oder Geräte verstanden. Vgl. zum medienhistorischen Verhältnis im Kontext der Begriffe analog/digital Manovich (2001).

16 Philip Rosen hat dies auch als „digital mimicry bezeichnet“, die - und dieser Aspekt wird in den nachfolgenden Überlegungen noch weiter Thema sein - in ihrer notwendigen Bezogenheit auf ,ältere“ Medien „temporal or historiographic conflations“ hervorbringe (Rosen 2001: 314-31). 
Hartmut Winkler (1997) hat in Docuverse angesichts der Hysterie um Multimedia und der Computer-Faszination Einspruch gegen die affirmative ,Rhetorik des Neuen' erhoben, Lev Manovichs Kritik am Mythos des Digitalen führt ihn zur Vermeidung des Ausdrucks digital und zum - freilich kaum minder strahlenden Begriff New Media (Distelmeyer 2012: 176, Hervorh. FH).

Darüber hinaus hat die Vorstellung Konjunktur, dass - in Abgrenzung zum akzidentiellen Verhältnis von digitalen Informationen und Wahrnehmungsgegenstand - das Analoge ,natürlicher' und wirklichkeitsnäher sei, weil etwa bei der fotochemischen Aufnahme noch ein indexikalischer Bezug zur und eine Interaktion mit der profilmischen Wirklichkeit vorliegt. Das Verhältnis zwischen Wahrnehmungsgegenstand und analogem Aufnahmeverfahren wird aus diesem Grunde als dem Menschen näher, als natürlicher empfunden, da es angeblich noch auf einer direkten Beziehung beruht und damit auch der menschlichen Wahrnehmung näher ist. Im Verlauf dieser Studie wird gezeigt werden, wie sich dieses Image als Mythos auf die Vermittlung von Bildern auswirkt, die analoge Filmgeschichte konnotieren.

Die Konnotation, dass analoge Medientechnik einen näheren Bezug zur Wirklichkeit zeitigt, ist vor allem differenziell mit der Vorstellung von digitaler Dematerialisierung und in der Folge dann auch mit Blick auf die Zuschreibung von freier Zirkulation zu sehen. Im Kontext der angenommenen digitalen Zirkulations- und damit omnipräsenten Zugangsmöglichkeiten reiht sich ein weiterer Mythos ein, der mit der numerischen Qualität einhergeht: die Vorstellung der endlosen, verlustlosen Kopierbarkeit der Informationen (Flückiger 2008: 45). Dass dem nicht voraussetzungslos so ist, sondern dass auch hier das mediale wie soziokulturelle Milieu der Manifestation eines Phänomens mitberücksichtigt werden muss, wird in der Literatur deutlich (siehe aus technischer Perspektive das Problemfeld von Konsistenz von Informationen in verschiedenen Systemen). ${ }^{17}$

Digitalisate - etwa von analogen Filmen - können in unterschiedlichsten Formen tatsächlich als Zugangselemente dienen und damit das materielle, fotochemische historische Artefakt des analogen Filmstreifens aus der Gebrauchskette (etwa als Projektionselement) nehmen und deshalb schonen. Hier zeigen sich die besonderen Vorteile einer digitalen Dematerialisierung.

17 Zur irrigen Vorstellung von allumfassender digitaler Verfügbarkeit bei gleichbleibender Qualität: „[Es] müssen digitale Bilddaten bis heute in den meisten Fällen wegen der hohen Datenmengen komprimiert werden, sodass auch die Kompressionsalgorithmen über mehrere Generationen immer deutlicher Fehler nach sich ziehen [können]“ (Flückiger 2008: 46). Flückiger zitiert pointiert Coy (2000): „Eine völlig fehlerfreie (also beliebig korrigierbare) Kodierung ist auch mit digitalen Systemen niemals erreichbar“ (Coy, zit. n. Flückiger 2008: 46). 
Zudem wird durch digitale Dispositive als Distributions- wie Zugangsmöglichkeiten ein räumlich diversifizierter Zugriff (etwa über das Internet) auf Digitalisate grundsätzlich eröffnet.

Dennoch darf dies nicht darüber hinwegtäuschen, dass hier in mehrfacher Hinsicht Abbildungsprozesse wirksam werden, die formierend auf das erscheinende Material Einfluss nehmen. Dies kann von Fragen, wie das Digitalisat überhaupt entstanden ist (Scanprozess), über die Frage, ob es in der Postproduktion bearbeitet wurde (Imaging, Color Grading/Lichtbestimmung, neue Rahmungen und Zuschneidungen des Bildformats in Form von Cropping etc.) und inwieweit die Bilder komprimiert wurden, bis hin zur dispositivischen Anordnung gehen: Sehe ich den entsprechenden Film ohne eindeutige Quellenangaben auf YouTube oder auf einer kuratierten, mit paratextueller Rahmung versehenen Archivseite?18 Eindrücke von freier Zirkulation und des immer möglichen Zugriffs verschleiern auch technisch bedingte Modifikationen des Materials. Zugleich verstellt die Vorstellung der potenziell unendlichen (verlustlosen) Kopierbarkeit auch den Blick auf weitere kontextuelle und mediale Formierungen der Wahrnehmung der Bilder.

Flückiger spricht mit Blick auf die zunächst technisch ermöglichte Zirkulation von einem „digitalen Ökosystem“ als Tauschsystem. Damit verbindet sie einen weiteren Aspekt des Zusammenwirkens von polymorphen technischenVoraussetzungen und deren praktischen Konsequenzen mit soziokulturellen, auch ideologisch wirksamen Überformungen - in diesem Fall der engen Verbindungen von digitaler Zirkulation, Waren- und Wertproduktion auf einem globalen (Kommunikations-)Markt:

Da die digitalen Daten in abstrakter mathematischer Form vorliegen, sind sie [...] Teil eines digitalen Ökosystems und eignen sich besonders für die Transmission, die Einspeisung in verschiedene Mediensysteme, und die Transformation, die Bildbearbeitung oder Wandlung (z. B. Bild in Ton). Damit sind wir wieder bei der Metapher vom Tauschsystem. Bildlich könnte man anführen, dass die binäre Kodierung wie eine universelle Währung funktioniert, welche globale Kommunikation erleichtert (Flückiger 2008: 47).

Der Bildkulturwissenschaftler Tom Holert hat die enge Verbindung von digital und dem globalen Markt ideologiekritisch auf der Bildebene untersucht,

18 Insbesondere in der Digitalisierung und Sicherung von analogen Filmen wird die Debatte um unterschiedliche Kompressionsformen heftig geführt (Speicherung ohne vs. mit Verlust). Zum hier anzuwendenden Konzept der archival pragmatics, die unterschiedliche Kompressionsformen in ihrem pragmatischen Zusammenhang modellieren, vgl. Flückiger/Heller/Op den Kamp et. al. (2016). Zu Standardisierungen in der digitalen Langzeitsicherung auch digitalisierter Filme vgl. F. Heller (2017). 
indem er an Manovichs Ansatz, Mythen des Digitalen zu dekonstruieren, anknüpft. Holert begreift insofern ,Mythos' als ein Bildkonzept, welches durch seine überzeitlich fixierende Form der Sinnproduktion ein nachhaltiges Versprechen auf Evidenz vermittelt. Unter diesem Vorzeichen analysiert Holert weit verbreitete Icons und Bilder der Digitalisierung als Elemente semiotischer Codes (u. a. etwa das Icon des Microsoft Internet Explorers). So leitet er die konzeptuelle Verbindung von digitalen Technologien und Kommunikationsformen her - als dematerialisierendes Abbruchunternehmen, das ,alte Kommunikationsmodelle niederreißt (Holert 2002). Vor diesem Hintergrund modelliert er den Neologismus der (Globo-)Digitalizität, der auf das Prinzip eines den Globus umfassenden Raumkonzeptes verweist, welches gleichzeitig durch allgegenwärtige Kommunikation kontrolliert wird (Holert 2002).

Holerts Thesen sind für meine weiteren Überlegungen insofern interessant, als er über das Bildkonzept im Modus des Mythischen das Evidenzversprechen ableitet: Die Mystifizierung von Images (FH) des Digitalen habe zur Folge, dass man den Eindruck bekomme, die Dinge seien allein aus sich heraus bedeutungsvoll. Wenn man Digitalizität (Holert 2002) untersuche, dann gehe es um den Nachvollzug und die kritische Reflexion einer „euphorischen“ Evidenz der Bilder von Digitalität und deren räumlichen Pendants des globalen Marktes (Holert 2002).

Als kurzes Zwischenfazit lässt sich auf der systematischen Ebene festhalten: Die digitale Domäne, insbesondere in Form von digitalen Bildern und in medialen Praktiken ihrer Zirkulation und Rezeption, weist technische Voraussetzungen auf, die im soziokulturellen Kontext der ersten 15 Jahre des 21. Jahrhunderts spezifische diskursive Images und technoimaginäre Topoi ausgebildet haben, welche auf kommende Nutzungsformen als zukunftsorientierte Utopien von Potenzialen der Technologien projektiv wirken.

Die sich in ästhetischen und distributiven Praktiken manifestierende Imagologie digitaler Bilder beruht im Wesentlichen auf fünf Grundeigenschaften, wie sie von Manovich ausformuliert, von Flückiger ergänzt und dann unter anderem von Distelmeyer aufgegriffen und kommentiert wurden. Diese Grundeigenschaften zeitigen jeweils Konsequenzen in weiterführenden Diskursen und medialen Praktiken.

- Programmierbarkeit: Die Programmierbarkeit digitaler Daten bezeichnet ein Set von Handlungsanweisungen, die auf die Ausgangsdaten angewandt werden sollen (zum Beispiel Formen der Bildbearbeitung) oder durch welche zwischen verschiedenen Daten neue Beziehungen geschaffen werden, etwa durch Hyperlinks, Montage und Compositing.

- Modularität: Das digitale Bild kann aus verschiedenen Bildteilen oder Bildschichten bestehen, zwischen denen komplexe räumliche oder zeitliche 
Beziehungen definiert werden. Distelmeyer benennt mit Rekurs auf Manovich die zugrunde liegende Computerlogik als „Datenbank-Logik“ (Distelmeyer 2012: 111). Diese bedeute keineswegs nur ein gleichberechtigtes Ordnungsprinzip unter vielen, sondern eine symbolische Form, die anstelle des Zusammenhangs das Auswahlprinzip stark mache. Die „Logik des Digitalen“ sei, so Winkler, „eine Logik der Auswahl“, die einer konkurrierenden Logik, jener der syntagmatischen Reihung eben, gegenüberstehe (Winkler, n. Distelmeyer 2012: 111).

- Direktzugriff (Random Access): Durch ihre mathematisch definierte Form lassen sich digitale Daten direkt adressieren. Random Access gilt als Ursprung der Auflösung von linearen Denkmustern sowie der Bildung von netzwerkartigen Strukturen, wie sie sich durch Hypertexte mit zahlreichen Links realisieren ließen, so Flückiger mit Rekurs auf Flusser, der dies als neue Form des nichtlinearen, assoziativen Denkens liest (Flückiger 2008: 49).

- Interaktivität: Durch Programmierbarkeit und den Direktzugriff eignen sich digitale Daten besonders für interaktive Kommunikation mit dem Rezipienten beziehungsweise Nutzer/User (Flückiger 2008: 50).

Am Beispiel der zugeschriebenen interaktiven Qualität digitaler Medien wird Manovichs Ansatz, Mythen des Digitalen mit ihren kulturellideologischen Implikationen zu perspektivieren, besonders deutlich: In seinem viel zitierten Kapitel "The Myth of Interactivity“ (2001: 55 ff.) zieht Manovich zunächst eine historische Linie zu Vorgängermedien. Er zeigt, dass die viel gepriesene scheinbar ,neue computerbasierte Interaktivität in der Tradition einer massenmedialen Moderne steht, die mentales Leben externalisieren will: „The literal interpretation of interactivity is just the latest example of a larger modern trend to externalize mental life, a process in which media technologies - photographies, film, VR - have played a key role" (Manovich 2001: 56). Manovich ordnet diese Entwicklung einer modernen Sehnsucht der Massengesellschaft nach Regulierung des Privaten über die Vernetzung mit dem öffentlich Geteilten zu (Manovich 2001: 6o). ${ }^{19}$ Er zeigt, dass Interaktivität nicht nur eine Form der (freien) Teilhabe an kulturellen Phänomenen ist, sondern auch eine Form der Regulierung. Eine (Hyper-)Link-Struktur lässt uns zwar vordergründig die Wahl über den $\mathrm{zu}$ folgenden Pfad, der sich alinear präsentiert und dessen Entfaltung von unserer interaktiven Handlung mit dem Medium abhängt; dennoch steht dahinter eine vorprogrammierte Struktur: „In short, we are asked to follow

19 „Hence the objectification of internal, private mental processes, and their equation with external visual forms that can easily be manipulated, mass produced and standardized on their own. The private and the public become regulated“ (Manovich 2001: 60). 
pre-programmed, objectively existing associations" (Manovich 2001: 61). Insofern steht hinter dem Image von Interaktivität ein komplexes Wechselspiel aus Internalisierung äußerer Strukturen und umgekehrt: einer teilenden Veräußerung innerer Prozesse - dies im Modus einer alinearen Struktur, welche Nähe zum Assoziativen aufweist. Knotenpunkt der assoziativen Struktur ist die Eigenschaft digitaler Daten zu einem Random Access.

Vor dem Hintergrund des Austauschverhältnisses zwischen Innerem und Äußerem ist es wichtig, in den folgenden Fallstudien zu beachten, wann welches Image einer Technologie, welche technische Voraussetzung wann in welcher Form wirkungsästhetisch zum Ausdruck kommt.

- Versatilität (Distelmeyer 2012: 111) bezeichnet die Eigenschaft der Variabilität eines Objekts der ,Neuen Medien'. ,Neue Medien` und ihre Phänomene demonstrieren eine charakteristische Instabilität. Distelmeyer macht die Variabilität an der Modularität und numerischen Repräsentation fest. So fungiert die Eigenschaft zugleich als Versprechen: „Ein Objekt der Neuen Medien (zum Beispiel Film auf DVD oder BD) ist nichts ein für allemal Festgelegtes, sondern etwas, das in den unterschiedlichen, potenziell unendlich vielen Versionen existiert“ (Manovich 2001, zit. n. Distelmeyer 2012: 111). Damit geht auch die Trennung zwischen dem Zugangs-Interface und der zugrunde liegenden, nicht direkt für den Menschen sichtbaren Datenform einher. Hier greift die Unterscheidung der - von Distelmeyer so benannten - „Computer-Ebene“ von der semiotisch und damit auch kulturell codierten Ebene des Zugriffs auf das digitalisierte Material (vgl. insbesondere Menü-Interfaces bei Blu-Ray-Discs respektive Streaminganbietern). Diese Unterscheidung basiert auf dem Problem der Opazität digitaler Daten; dem Phänomen, dass digitale Codes per se nicht für den Menschen wahrnehmbar sind, sondern immer schon eine Umsetzung in einer Darstellung benötigen.

Wie mehrfach hervorgehoben, untersuche ich, wann und unter welchen Bedingungen technologisch gegebene Möglichkeiten in einer bestimmten Perspektive und Kontextualisierung, in einem bestimmten medialen Milieu (Schneider 2012: 100), zum Ausdruck kommen - wobei Ausdruck wiederum als ein zeiträumlicher medialer Vermittlungsakt zu begreifen ist. Die Grundeigenschaften von digitalen Bildern - gerade mit ihrer Versatilität und Flexibilität - leisten vielfältigen diskursiven, ideologischen Formierungsmöglichkeiten Vorschub; dies sowohl auf der Ebene der Ästhetik als auch auf der der Zugangsform. Deshalb lassen sich bei der Analyse der konkreten, sich situativ entfaltenden medialen Manifestationen die impliziten Geschichtsbildmodellierungen vor allem in der wirkungsästhetischen Dimension herauspräparieren. Dabei bedeutet der enge diskursive Zusammenhang von (Waren-) 
Tauschsystemen und Zirkulation mit digital eine wichtige Engführung der Perspektive auf die Digitalisierung von Filmbildern.

\subsection{Film im Zeichen von Digitalisierung}

Grundsätzlich hat die Entwicklung digitaler Medientechnologien das Medium Film auf fast allen Ebenen der Produktion sowie in den Formen der Rezeption verändert. ${ }^{20} \mathrm{Im}$ Wesentlichen zusammengefasst betrifft dies die folgenden Ebenen:

- Die Aufnahmetechnik der Filme wurde im Verlauf der 2oooer Jahre weitgehend digital: Die verwendeten Kameras fixieren die einfallenden (Licht-)Informationen nicht mehr mittels der Interaktion mit einem fotochemischen Filmstreifen, sondern die eingehenden Informationen werden nun über einen Sensor aufgenommen und über einen Chip in der Kamera in binäre, digitale Daten umgewandelt.

- Die Postproduktion wurde ebenfalls in die digitale Domäne überführt. Zunächst geschah dies noch in Form des Digital Intermediate Process (DIProzess): Ein noch analoger, belichteter Filmstreifen wird gescannt, das heißt in die digitale Domäne überführt. Damit stehen in der Postproduktion alle Bildinformationen unter der Maßgabe der polymorphen Eigenschaften von digitalen Bildern - etwa Modularität und Programmierbarkeit - der Bearbeitung zur Verfügung. Dies kann die Lichtbestimmung, das Color Timing beziehungsweise Grading, betreffen (,Pionier-Produktionen waren hier O Brother, Where Art thou [200o] und die ersten Lord-OF-THERINGs-Filme [2001-2003]). Auch das Einfügen beziehungsweise Kreieren von Visual Effects erreicht neue Dimensionen (vgl. ausführlich und differenziert zu den unterschiedlichen Formen Flückiger 2008). ${ }^{21}$ Letztendlich stehen in der digitalen Domäne die Informationen für jegliche Eingriffe zur Verfügung. Zunächst wurden noch die im DI-Prozess bearbeiteten Filme wieder auf Filmstreifen zur Kinodistribution ausbelichtet. Da sich, wie unten erläutert, auch die Projektionstechnik in den Kinosälen in die digitale Domäne verlagert hat, werden die Filme für die Kinodistribution mittlerweile in einer digitalen Form belassen und standardisiert digital für die

20 An dieser Stelle sei ausdrücklich darauf hingewiesen, dass sich die publizistische und wissenschaftliche Debatte meist auf die visuelle Ebene von Filmbildern bezieht. Der Filmton war bereits seit Anfang der 1990er Jahre digital.

21 So muss man die Begrifflichkeit präzisieren, da es gilt, vor allem zwischen Special Effects und digitalen Visual Effects wie computergenerierten Bildern (CGI) zu unterscheiden (Flückiger 2008: 22-25). 
Kinodistribution als Digital Cinema Package (DCP) komprimiert und verpackt. Dies verändert in der Medientransition der 2oooer Jahre ganze Distributionsworkflows, etwa Sicherheitsmaßnahmen gegen Piraterie oder die Gestaltung der Transportwege, da die Filmrollen nicht mehr als materielle Objekte an die Aufführungsstätten versendet werden müssen.

- Die Filmdistribution erfolgt mittlerweile - noch differenzierter, als es in der analogen Ära der Fall war (auch hier existierten schon Versionen für die Projektion zu Hause, etwa in kleineren Filmformaten als $35 \mathrm{~mm}$ oder dann später in Form der VHS) - neben der Auswertung im Kino auf verschiedenen Trägern, in verschiedenen Formaten und Editionen für den Heimvideomarkt, für Fernsehen oder Streaming. ${ }^{22}$

Mit der Digitalisierung der Produktion und Distribution vervielfältigen sich nun die Möglichkeiten der Migration in unterschiedliche Dispositive. Da das Filmmaterial in der digitalen Domäne mittels unterschiedlicher Medien gespeichert werden kann und auf unterschiedlichen Wiedergabegeräten abspielbar ist, ergibt sich eine Vielzahl von Rezeptionsmöglichkeiten - jeweils mit spezifischen ästhetischen und diskursiven Formierungen; dies etwa in Form von Anpassungen für Screens, die unterschiedlich in den Alltag eingebunden sind und deshalb jeweils mit anderen Aufmerksamkeitsstrukturen im öffentlichen, aber auch im privaten Raum konkurrieren. Zugleich, dies kommt mit Blick auf die Variabilität der konkreten Ästhetik noch erschwerend hinzu, zeitigen die unterschiedlichen Sichtungsmöglichkeiten wie vor allem Fernsehbildschirme und Computer oft noch eine Vielzahl von Einstellungsmöglichkeiten und Parametern, die das erscheinende ästhetische Objekt unter Umständen nachhaltig prägen können. Von den Möglichkeiten der digitalen Edition und der entsprechenden paratextuellen, kontextualisierenden Präsentation wird im Verlauf der Studie noch ausführlich die Rede sein.

- Wie schon angesprochen, ist darüber hinaus auch die Projektion im Kino digital geworden. Insbesondere David Bordwell hat 2012 in seiner Studie Pandora's Digital Box. Films, Files and the Future of Movies die Konsequenzen dieser zunächst technologisch bedingten Entwicklung als Transformation der historisch gewachsenen kulturellen Praxis der Kinoprojektion untersucht.

22 Historisch ist eine ähnliche Entwicklung mit Blick auf Veränderungen von Filmen zu beobachten, die zunächst für die Kinoauswertung produziert wurden und dann für die Aufführung im Fernsehen angepasst wurden (vgl. dazu später insbesondere im vorliegenden $\rightarrow$ Kap. 2 den Abschnitt zur Colorization-Debatte). 
- Nicht zuletzt sei hier darauf hingewiesen, dass all die genannten Veränderungen in der Filmproduktion und -distribution nachhaltige Folgen für die Filmsicherung und -archivierung zeitigen. Filmarchive, die bis dato vornehmlich auf die Lagerung von fotochemischem Film und seinen nonfilmischen Paratexten und Ko-Texten (Kessler 2010) spezialisiert waren, müssen nun als sogenannte hybride Archive sowohl in der analogen als auch in der digitalen Domäne agieren und Sicherungskonzepte - insbesondere auch für digitale Daten in einer Langzeitperspektive - entwickeln (vgl. hierzu F. Heller 2017).

Schon in dieser kursorischen Aufstellung der Veränderungen im Horizont der Medientransition wird deutlich, wie sehr mit dem Medium Film verbundene Denkmuster mit digitalen Prozessen eine Re-Formulierung erfahren (müssen) - seien es etwa die Vorstellung vom Filmischen als Aufnahmeprozess oder die Konzeption des Kinodispositivs, dessen Bindung an die traditionelle öffentliche und soziale Institution Kino bereits mit dem Neologismus und Hybridbegriff Heimkino infrage gestellt wird.

Dennoch schreibt Ringler (2009) über die populäre Einschätzung der Digitalisierung des Films, dass diese vor allem über die filmische Wahrnehmungsebene des Zuschauers geprägt sei:

Der Begriff Digitalisierung hat sich in den letzten Jahren im Kontext der Filmindustrie Hollywoods zu einem mächtigen Schlagwort etabliert, welches sich zunächst nur schwer fassen lässt. Wer heute von Digitalisierung spricht, beschreibt zumeist nur das, was der Rezipient letzten Endes im Kino sieht, nämlich Special Effects (Ringler 2009: 13). ${ }^{23}$

Dies ist an dieser Stelle deshalb hervorzuheben, weil Film als illusionsbildendes und zugleich erzählendes Medium bei der Zukunftsphantasie digital/Digitalisierung eine zentrale Rolle spielt. Mit all den filmischen Möglichkeiten, Traum- und Phantasiewelten zu eröffnen, stellt das Medium Film in der heutigen Populärkultur eine fruchtbare Projektionsfläche dar: als tatsächlich projizierte Bewegtbilder, die zu Imaginationen, zur emotionalen Involvierung und zur illusionären Immersion seitens des Zuschauers einladen. Gleichzeitig sind die jeweiligen digitalen bild- und tongebenden Technologien und Wiedergabesysteme Gegenstand von populärkulturellen Projektionen, die sich zum Teil aus der ersten Ebene - der sensuellen Wahrnehmung von

23 So sehr Ringlers Befund in der Essenz für die populäre Wahrnehmung zutreffen mag, so muss man seine Begrifflichkeit präzisieren, da es gilt, vor allem zwischen Special Effects und digitalen Visual Effects wie computergenerierten Bildern (CGI) zu differenzieren (Flückiger 2008). 
Bewegtbildern - speisen. Wie schon in den obigen Ausführungen deutlich werden sollte, leisten die technologischen Voraussetzungen der digitalen Domäne solchen Projektionen Vorschub.

Illustrative Beispiele für die Überlagerung von fiktionalen, ja gar phantastischen Filmwelten und die Zuschreibung magischer Attribute an Entwicklungen der digitalen Filmtechnologie lieferten exemplarisch zu Beginn der 2oooer Jahre die schon zitierten Lord-of-The-RIngs-Filme. Die Einschätzung filmtechnologischer Entwicklung als "digital wizardry“ (Klinger 2006a: 122) verband sich als Technoimaginäres (H. Böhme 200o) ${ }^{24}$ in der Vermarktung und somit auch im populären Diskurs mit dem phantastischen Inhalt der Reihe. Die Diskussion wurde im Zusammenhang mit der Weiterführung des Franchisesystems der Новвіт-Filme fortgesetzt. So haben sich in den Jahren 2012 bis 2014 unter dem Stichwort Digitalisierung des Films die einschlägigen populären Debatten vor allem um die konkret sichtbaren und erfahrbaren Bildbeispiele aus dieser Reihe gedreht: etwa um die die menschliche Wahrnehmung herausfordernden Massenkampfszenen, die mit neuer Software der Bildgenerierung möglich wurden; die irrwitzigen Drachenangriffe, bei denen Bewegungen von Feuer und Luftzirkulationen filmbildlich simuliert und mit atmosphärischer Sogwirkung inszeniert wurden - das alles in 3-D-Technik und einer doppelten Bildgeschwindigkeit von 48 Bildern pro Sekunde. Doch insbesondere bei der Frage der Bildgeschwindigkeit fand sich Regisseur Peter Jackson im Zentrum der Kontroverse, die sich um die Frage des Verhältnisses zwischen sich verändernden, digitalen Bildästhetiken und Vorstellungen vom Filmischen, die historisch gewachsen sind, drehte: Nach der Kritik am ersten Teil der Новв тт-Reihe, der Film sehe in der neuen Bildgeschwindigkeit zu sehr nach Video- und Fernsehästhetik aus, versuchte Jackson nach eigener Aussage jedem darauffolgenden Teil „mehr Textur zu geben“ (Jackson, zit. n. Freeman 2014). Der von Jackson gebrauchte Begriff „Textur" des Films verweist unter anderem auf einen Look des Films, wie wir ihn aus der fotochemischen Ära, vor allem geprägt durch das Filmkorn, kennen,

24 Als Imagologie der Technik bezeichnet H. Böhme ein Forschungsfeld, das die „Leitbilder der Technik“ (Stöcklein 1969, n. H. Böhme 200o), die technischen Träume und Phantasien, das Wunderbare und Mythische, schließlich die technischen Utopien untersucht. Das Technoimaginäre ist für die Kulturgeschichte nicht nur aufschlussreich, weil in alten Gesellschaften technische Praktiken vielfach mit dem Sakralen und Religiösen, dem Phantastischen oder Traumhaften verbunden waren, sondern auch weil es - so ein Befund H. Böhmes - vor allem heute in allen Massenmedien Antrieb hat und Ausdruck findet (H. Böhme 200o: 4 FH. [Die Zitationsweise im Fließtext erfolgt auf der Grundlage des von der Onlinequelle erstellten Dokuments im PDF-Format. Insofern werden zur Präzisierung Seitenzahlen auf dieser Grundlage angegeben, allerdings in dieser Zählweise dann mit „FH“ gekennzeichnet]). 
dessen Simulation in der digitalen Domäne nun - bei aller Unbestimmtheit der Referenz von über 100 Jahren analog geprägter Ästhetikgeschichte - das Filmerlebnis als solches simulieren und wieder nobilitieren sollte. An dem Beispiel wird deutlich, wie eng sich tatsächliche technologische Veränderungen in der Produktion und Projektion mit der Rezeptionsebene, der ästhetischen Erfahrung eines spezifischen Filmes - mitsamt dessen erzählter Fiktion -, in überformenden Diskursen und Imaginationen von Technologien vermengen können. ${ }^{25}$ Dies ist aber nicht abzutrennen von historisch gewachsenen medialen Konditionierungen und Sehgewohnheiten, die die Bereitschaft des Zuschauers zur Immersion in die Fiktion als Erlebnis nachhaltig bedingen.

So stellt sich weitergehend die Frage, wie sich Vorstellungen des Labels digital filmisch beziehungsweise kinematografisch in Diskurskreisen und Nutzungsformen konstituieren. Die Debatten, wie sie exemplarisch anhand der Новвіт-Reihe illustriert wurden, zeigen sich in ihren Konfliktlinien und kulturellen Konsequenzen noch deutlicher in den Bereichen, in denen tatsächlich analoge beziehungsweise historische Filme im Kontext digitaler Technologien und Medienumgebungen (interessegeleitet) wieder aufgenommen werden. (Dies werde ich in der Folge als Praktiken der Reprise bezeichnen in Anlehnung an das Konzept des Filmwissenschaftlers François Niney zur Wiederaufnahme von Archivbildern: vgl. das Zwischenfazit $\rightarrow$ Kap. 3.9).

Hier artikulieren sich pragmatische Zusammenhänge, in denen die wechselseitige Abhängigkeit des historisch gewachsenen, mit der Erfahrung der Moderne verwobenen Mediums Film und digitalen Technologien besonders deutlich wird. Bei der digitalen Wiederveröffentlichung von historischen Filmen entstehen auf der Wirkungsebene sinnliche Konfigurationen, die

25 Damit verfolge ich einen Ansatz, der Annette Kuhns methodische Vorüberlegungen (2002) aufgreift, welche sie in ihrer ethnohistorischen Studie zum Zusammenhang von kulturellem Gedächtnis, Kino, Film und populärer Kultur formuliert hat. Kuhn kritisiert den oft in medienwissenschaftlichen Studien zu findenden Dualismus, entweder eine Filmtextanalyse ohne hinreichende soziokulturelle Rückversicherung durchzuführen oder aber soziokulturelle Erhebungen vorzunehmen, ohne der ästhetisch-textuellen Ebene gerecht zu werden: „This division [...] produces a conceptual and methodological dualism of text and context - a divorcing of film texts from their industrial, cultural and historical contexts, and vice versa, and this weakens studies of cinema and other media by ensuring that accounts of media texts and their consumption and reception remain incomplete" (Kuhn 2002:4). In der vorliegenden Studie betrifft dieses methodische Problem das Verhältnis zwischen ästhetischen Praktiken (und ihrer Analyse) und der Erhebung und Auswertung soziokulturell funktionaler Vorstellungen von digitalen Technologien. 
unser Erleben von Zeit - Gegenwart und Vergangenheit ${ }^{26}$ - unter dem Vorzeichen Film und Filmerlebnis modellieren. ${ }^{27}$

Dabei ist zu beachten, dass der Begriff digital, wie auch in $\rightarrow$ Kap. 1 einleitend konzeptualisiert, höchst zeit- und kontextgebunden zu verstehen ist: Angesichts der schnelllebigen Entwicklung im Bereich der digitalen Technologien befinden sich die technischen Standards und die Möglichkeiten der Datenverarbeitung wie der Bildbearbeitung in einem rasanten Wandel. Umso mehr muss die Frage Was ist Digitalisierung? abgewandelt werden zu Wann ist Digitalisierung? ${ }^{28}$

Eine ähnliche pragmatisch orientierte Perspektive ergibt sich auch in Hinblick auf die Frage nach der Referenz auf das historische Medium Film. Dies wird besonders deutlich, wenn man zentrale Werke zu dem Problemkomplex von Autoren wie David N. Rodowick und Giovanna Fossati betrachtet. Wenn man Film digitalisiert, muss man sich zuvorderst fragen, was man unter dem Objekt des Transfers, unter Film, versteht. So nimmt vor allem in Fossatis Studie From Grain to Pixel. The Archival Life of Film in Transition (2009) die Bestimmung von Film in einer pragmatischen Perspektive durch unterschiedliche Frameworks (Film „as Original“, „as Art“, „as Dispositif“, „as State of the Art“) einen breiten Raum ein (Fossati 2009: 117-131). Die das Verständnis von Film bestimmenden Frameworks bedingen - so Fossatis Ansatz - maßgeblich die archivarischen Praktiken beziehungsweise die Anwendungsformen von digitalen Technologien in der Filmsicherung, -restaurierung und -rekonstruktion. Fossati bezieht sich hier, wie es sich dann später in der Rezeption auch bei Flückiger unter anderem 2012 und 2018 fortsetzt, besonders auf Überlegungen aus David N. Rodowicks The Virtual Life of Film (2007), der André Bazins berühmte Frage: „What is cinema?" umformuliert in "What was cinema?". Die von Rodowick programmatisch gewählte Vergangenheitsform weist auf die historisierenden Kontexte beziehungsweise die zeitliche Abhängigkeit des jeweiligen Verständnisses und der Praktiken hin, in denen jeweils Cinema beziehungsweise Film verstanden werden können.

26 Vgl. auch zum grundsätzlichen Ansatz die Ausführungen von Koselleck (1989) $\rightarrow$ Kap. 1.

27 Hier zeigt sich eine Form der - später noch begrifflich präziser entwickelten - medial aisthetischen Historiografie, die unseren Alltag durchdringt; dies in einem Wechselspiel zwischen massenmedialer Öffentlichkeit und individueller Aneignung im Zeichen unterschiedlicher digitaler Dispositive.

28 Dies kann als eine erweiterte Auslegung des berühmt gewordenen Ausspruchs „Wann ist Kunst?“ von Nelson Goodman (1997/1968) angesehen werden. In diesem Zusammenhang werden symbolische Funktionen und Rezeptionsweisen in ihrer Zeitgebundenheit gesehen, was mithin jeweils die Bestimmung des Kunst-Status beeinflusst. 
Flückiger (2012: 3 f.) hat die vor allem in der Archiv-Community geführte Debatte um das Verständnis von Film rekapituliert und systematisiert, um die Referenzrahmen der Transition von historischem Material in die digitale Domäne beschreibbar zu machen: Film könne verstanden werden als historisches, materielles Artefakt (Filmstreifen) (Cherchi Usai 2000), als Performance (als dispositivisch geprägtes Projektions- und Aufführungsereignis), als konzeptuelle Gestalt (Carroll 1996) und vor allem als Text - also im Hinblick auf seinen Inhalt, seine ästhetische Struktur (ästhetische Imago nach Brandi 1963 beziehungsweise Janis 2005). Fossati fasst die möglichen Perspektiven wie folgt: „The material film artifact is typically the film preserved by the archivist, whereas the conceptual film artifact refers to its abstraction as an historical and aesthetic object" (Fossati 2009: 105). Die konkreten Gebrauchs- und Nutzungszusammenhänge bestimmen, welches Verständnis von Film jeweils zur Anwendung kommt. ${ }^{29}$ Flückiger/Heller/Op den Kamp et al. (2016) bezeichnen und konzeptualisieren dies mit dem Begriff "archival pragmatics.“

So macht insbesondere Fossati deutlich, wie sehr die pragmatischen $\mathrm{Zu}$ sammenhänge sogar definieren, was man als das eigentliche archivarische (materielle) Objekt begreift. Restaurierung und Sicherung arbeiten mit dem materiellen Artefakt Film, während andere archivarische Aufgaben wie etwa das Ausstellen (exhibition) und die Präsentation (presentation) eher auf der phänomenalen Ebene der Imago wirken. Insbesondere die beiden letztgenannten Aspekte haben nachhaltigen Einfluss auf die Ästhetik und auf die Wahrnehmung von Filmgeschichte (Fossati 2009: 107). ${ }^{30}$ Bei digitalen Bildern zeigt sich der Ansatz von "archival pragmatics“ noch deutlicher, da - wie Flückiger/Heller/Op den Kamp et al. (2016) gezeigt haben - aufgrund des nicht zwingenden Zusammenhangs von Code und Erscheinungsform bei digitalen Bildern je nach pragmatischem und archivarischem Kontext und Interesse zwischen dem Bild als information (oben auch „Ebene des Codes“ genannt) und der Ebene der appearance unterschieden werden müsse.

29 So findet sich zum Beispiel auch im Diskurs über das filmische Original die Spannung zwischen materiellem und konzeptuellem Artefakt: „The original can indeed be one of the possible conceptual artifacts (e. g. the director's cut or the film shown to the audience) or one of the possible material artifacts (e. g. the original camera negative or the only existing fragment of a projection print recovered by the archive)“(Fossati 2009: 106-107).

30 Sie verfährt damit differenzierter als Carroll, von dem sich Flückiger 2012 mit ihrer Hervorhebung des besonderen Verhältnisses von Trägermaterial der Informationen und der dadurch entstehenden Ästhetik der Bilder noch einmal distanziert: „While digitisation in accordance with Carroll's observation does not affect film as a text, it transforms the film as a material object and as a performative instance - not only on the level of its physical composition, but also in the sensory domain that affects the aesthetic dimension. Each of these modes of being, or ontologies, has a different history“ (Flückiger 2012: 5). 
Mein Erkenntnisinteresse zielt allerdings weniger auf die Analyse archivarischer Praktiken von konkreten Institutionen. Vielmehr geht es um die breitenwirksamen, erlebbaren Effekte von Filmen, die im Zeichen einer marktorientierten Zirkulation und im Kontext einer Imagologie der digitalen Domäne zu sehen sind; beides zeitigt Auswirkungen auf mediale Geschichtsbildmodellierungen.

Abschließend sei vor diesem Hintergrund zur Illustration ein Beispiel zitiert, in dem deutlich wird, inwieweit sich die diversifizierte Zirkulation von Filmen jenseits des Kinos konkreten Restaurierungsentscheidungen einschreiben kann, die wiederum Einfluss auf die Erscheinungsweise und Ästhetik von Filmen haben. So äußerte Grover Crisp, Seniorvizepräsident für Asset Management, Film Restoration und Digital Mastering bei Sony Pictures Entertainment, die folgende Einschätzung auf die Interviewfrage, wie sehr die Entwicklung der Blu-Ray-Disc Filmrestaurierung beeinflusst habe:

Blu-ray is a wonderful format that allows the viewer to experience a film much closer to what the theatrical experience is like. The higher resolution in both picture and sound also means that we need to provide the best quality materials in order to work at its maximum. What this means for restoration is that we can now work to provide better materials than would have been necessary in the past. Blu-ray and restoration go really hand-in-hand in that regard (Crisp, zit. n. B. Hunt 2011).

Crisps Aussage deutetauf einen Kreislauf hin, in dem digitale Distributions- und Editionsmöglichkeiten Einfluss auf ästhetische Entscheidungen im digitalen Restaurierungsprozess haben können (genauso gilt dies auch umgekehrt in die andere Richtung, wenn Restaurierungsprozesse zum Beispiel Editionsformen prägen). Die jeweiligen Konsequenzen der ästhetischen Formierungen lassen sich nur noch situativ bestimmen: So gilt es nicht nur danach zu fragen, was jeweils konzeptuell unter Film verstanden wird, sondern auch wie sich dies im konkreten filmischen Phänomen und den Bedingungen seines Erscheinens, seinem Dispositiv, auf unser ästhetisches und zeiträumliches Erleben - die Erfahrung von Vergangenheit, Gegenwart und Zukunft - auswirkt.

\subsection{Digitalisierung als methodologische und medienhistoriografische Herausforderung}

In den folgenden Ausführungen wird der Begriff digital aus kulturwissenschaftlicher Perspektive im Horizont historiografischer Konzepte präzisiert. Dies führt zu methodologischen Überlegungen, die nicht abzulösen sind von 
fachpolitischen und disziplinären Debatten, die sich in der Perspektivierung des Verhältnisses von Technologieentwicklung, Medienbegriff, Gesellschaft, Vorstellungen von historischer Dynamik und ästhetischen Strukturen manifestieren. Es geht zum einen um die konzeptuelle Fassbarkeit der Medientransition und die hier verhandelten Vorstellungen von Geschichtlichkeit; zum anderen ist dies im Zusammenhang mit der geschichtlichen Verortung der Arbeitsweisen von Medien- und Filmwissenschaft zu sehen.

Die Bezeichnung digital wird im Folgenden als ein "Set von Praktiken“ (Hediger 2005b) verstanden. Methodologisch wird dies in einer pragmatischen Herangehensweise umgesetzt. So bedeutet die Analyse der jeweiligen Praktiken die kritische Fokussierung auf konzeptuelle Muster, Konfigurationen des Technoimaginären und kulturelle Narrative, die den Anwendungsund Gebrauchsformen zugrunde liegen und in massenmedial verbreiteten ästhetischen Formen ihren Ausdruck und ihre Vermittlung finden. Die Formen des Gebrauchs interagieren mit Zuschreibungen an die Technik, denen von bestimmten technologischen Grundvoraussetzungen Vorschub geleistet wird. So ergibt sich eine Perspektive, in der digitalisierte Filme als ein komplexes Netz von Sinn- und Bedeutungsproduktionen verstanden werden. In diesem Netz wird das konkret sinnlich erfahrbare mediale Phänomen - inklusive seiner immersiv wirkenden Potenziale - als vielschichtige Ausdrucksform eines weiteren soziokulturellen Prozesses von Geschichtsbildmodellierung gesehen. Digitalisierte Filme werden vor diesem Hintergrund als ein clusterförmiges Medium impliziter aktueller Geschichtsvorstellungen begriffen. So weist Uricchio (2004: 24) darauf hin, dass Medien aus einer kulturwissenschaftlichen Sicht nicht nur Technologien, Institutionen oder Texte, sondern vor allem kulturelle Praktiken darstellen, die in einem weiteren gesellschaftlichen Kontext anzusiedeln sind.

Die einzelnen Faktoren und Praktiken innerhalb dieses Netzwerkes, die zu einem digitalisierten Film führen oder dessen Gebrauchsformen bestimmen, können ganz unterschiedlich gewichtet sein. Giovanna Fossati (2009) hat in ihrer Studie den theoretischen Zugang über eine Anverwandlung der sogenannten SCOT-Theorie - Social Construction of Technology - gewählt, um die archivarischen Praktiken unter dem Einfluss der digitalen Technologien konzeptualisieren zu können. Fossati legt einen besonderen Fokus auf die beteiligten Akteure. Sie betrachtet die Anwendung digitaler Technologien als im höchsten Masse abhängig von "major players“ im filmarchivarischen Feld (Fossati 2009: 22), welches insgesamt von einer institutionellen sowie interdisziplinären Heterogenität und Vielschichtigkeit von Interessen geprägt sei: „SCOT is a suitable instrument for studying a transitional process at the same time influenced by and influencing a large number of players 
and cutting across various layers (technological, economical, social, cultural, etc.)“ (Fossati 2009: 22). Interessanterweise identifiziert Fossati neben vielen anderen Akteuren im filmarchivarischen Feld ${ }^{31}$ insbesondere die wachsende Öffentlichkeit von „digital film users“ als einen an Bedeutung gewinnenden Faktor in diesem Netzwerk - weg von einem elitären, cinephilen Expertennischenpublikum (Fossati 2009: 151). Fossati begreift technologische Entwicklung vor allem als einen sozialen Prozess und nicht als ein sich autonom entfaltendes Vorkommnis („autonomous occurrence“, Bijker 1997, zit. n. Fossati 2oog: 22, 262, 151).

Die Frage, wie technologischer Fortschritt im Verhältnis zum Menschen und dessen sozialem Umfeld einzuschätzen sei, weist in der deutschsprachigen Medien- und Filmwissenschaft zu Beginn der 2oooer Jahre nicht nur eine geschichtstheoretische Dimension auf, sondern auch eine disziplinäre: so etwa in dem ,Selbstverständigungsband', welcher dem „Diskurs der methodischen und gegenständlichen Selbstreflexion“ verschrieben ist (H.-B. Heller/Kraus/ Meder et al. 2000: 7). Die Autoren sehen mit Blick auf Entwicklungen in den späten 199oer Jahren die dringende Notwendigkeit einer methodologischen Reflexion des Medienbegriffs und der damit einhergehenden analytischen Modelle des Medienwandels:

Die digitale Revolution zeitigt nicht allein neue Praxen, sie zwingt zugleich eine an den traditionellen Leitmedien Film und Fernsehen entwickelte Theorie und Analyse zur kritischen Selbstreflexion, mindestens aber zur Relativierung ihres Medienbegriffs (H.-B. Heller/Kraus/Meder et al. 2000: 7).

Anlässlich dieses Befundes diskutiert Hartmut Winkler methodische Tendenzen in der deutschen Medienwissenschaft mit Blick auf die jeweilige Perspektivierung des Verhältnisses von soziokulturellen Bedürfnissen der Menschen, von Gebrauchsformen und medientechnologischen Entwicklungen. Letztendlich, so Winkler, gehe es um die Frage, ob Technik in der Medienwissenschaft als Voraussetzung oder als Resultat gewichtet, gedacht

$31 \quad$ Fossati differenziert hier Filmarchive, die kommerzielle Filmindustrie, Politiker und politische Akteure im Kultursektor; dies sowohl national wie international: Sie nennt hier die EU und auf globaler Ebene die UNESCO; darüber hinaus spielen von der Industrieseite Hardware- und Softwareentwickler, Hersteller und Verbraucher von (fotochemischen) Filmmaterialien, von Laborequipment sowie die Labore selbst eine wichtige Rolle in dem Netzwerk. Hinzu kämen noch verschiedene Interessengruppen wie etwa die Digital Cinema Initative und das European Digital Cinema Forum sowie Fernsehsender, die Archivmaterial verstärkt ausstrahlen - wie zum Beispiel in Europa ARTE (Fossati 2009: 151). 
und behandelt werde, von welchem Ort und von welcher Ausgangssituation her kommend man Mediengeschichte schreibe (Winkler 2000: 11).

Eine exponierte Position der deutschsprachigen Medienwissenschaft paraphrasierend, beginnt Winkler mit Rekurs auf Friedrich Kittler und dessen Diktum „Medien bestimmen unsere Lage“ (Winkler 2000: 10). Winkler fasst in der Auseinandersetzung mit Kittler fachpolitische und methodologische Konsequenzen wie folgt zusammen:

Es wird [seitens der Kittler-Schule, FH] als eine kopernikanische Wende angesehen - und als Initialzündung der Medienwissenschaft selbst - den Blick umorientiert zu haben von der Ebene der Inhalte und der künstlerischen Formen auf jene Techniken, die keineswegs nur,Werkzeug' oder ,Voraussetzung kommunikativer Prozesse sind (Winkler 2000: 11).

Diesem technisch oft deterministisch argumentierenden Strang wird die Ausrichtung auf eine einseitig kausale Wirkung der Technik auf den sozialen Prozess vorgeworfen. Darüber hinaus zitiert Winkler einen weiteren Kritikpunkt: Solche Diskurse neigten zur Fetischisierung - Fetischisierung verstanden im Marx'schen Sinne, dass nämlich damit die fertige Ware den Prozess ihrer Hervorbringung verdecke (Winkler 2000: 13).

Im Gegensatz dazu beschreibt Winkler Alternativansätze, die ,anthropologisch' genannt werden, weil sie ,am Menschen' als Subjekt der Mediengeschichte festhalten und in den menschlichen Praktiken den Motor der Entwicklung sehen. Anthropologisch' seien in diesem Sinne nahezu alle Medientheorien bis zum historischen Bruch bei McLuhan, der neben Walter Benjamin als Hauptzeuge der theoretischen Wende in Anspruch genommen werde: „All diesen [anthropologischen, FH] Ansätzen ist gemeinsam, daß sie ein medientechnisches ,Apriori kultureller Prozesse vehement bestreiten würden“ (Winkler 2000: 12). Der anthropologisch argumentierende Strang 32 erfragt von einer existierenden Technik, "was diese in die Welt gebracht hat" (Winkler 2000: 13). Technik wird hier nicht als Ausgangspunkt begriffen, sondern als Resultat von sozialen und kulturellen Praktiken; die Hervorbringung von Technik wird nicht einzelnen Erfindern, sondern einem größeren gesellschaftlichen Entwicklungsprozess zugeschrieben (Winkler 2000: 14).

Angesichts der skizzierten Dichotomie schlägt Winkler ein Modell der zyklischen Einschreibung vor:

32 Unter, anthropologisch' seien auch jene elaborierteren Modelle zu fassen, die von mentalitätsgeschichtlichen oder wunschtheoretischen Prämissen ausgehen (Winkler 2000: 12). 
Technik ist das Resultat von Praxen, die in der Technik ihren materiellen Niederschlag finden; Praxen (einige, nicht alle Praxen!) schlagen um in Technik, dies wäre die erste Phase des Zyklus. Und gleichzeitig gilt das Gegenteil: Dieselbe Technik ist Ausgangspunkt wiederum für alle nachfolgenden Praxen, indem sie den Raum definiert, in dem diese Praxen sich ereignen. Dies ist die zweite Phase des Zyklus. Einschreibung der Praxen in die Technik und Zurückschreiben der Technik in die Praxen (Winkler 2000: 14).

Damit seien „Diachronie und Synchronie, Diskurs und Struktur [...] auf systematische Weise miteinander verbunden“ (Winkler 2000: 15). ${ }^{33}$ Dieser Befund lässt sich produktiv auf unseren Gegenstandsbereich übertragen: Auf der einen Seite stehen im Kontext von digitalisierten Filmen die soziokulturellen Bedürfnisse - etwa des Zugangs -, die digitalen Technologien in Bezug auf Film ihren Wert geben. Dies ist allerdings nicht abgetrennt zu sehen von der vorangegangenen (analogen) Film- und Mediengeschichte, die eine entscheidende Rolle für die Entwicklung von Ansprüchen und Bedürfnissen etwa angesichts von medialen Erlebnisformen spielen. Jene können wiederum von digitalen Technologien aufgegriffen und bedient werden. So gilt auf der anderen Seite - mit Winkler gesprochen im zweiten Zyklus: Digitalisierte Filme und ihre Nutzungsformen schreiben sich wiederum in die Mediengeschichte ein und schaffen ihrerseits spezifische Bedingungen und Bedürfnisse - vor allem in Hinblick auf die Nutzung und Zuschreibung von Potenzialen von Medien. Digitalisierte Filme definieren darüber den sozialen und kulturellen Raum und Rahmen, in dem wir wiederum Film begreifen und behandeln - was seinerseits neue Sehsüchte nach medialen Erlebnisformen befördert. Mit Winkler (2000: 22) lässt sich somit kulturkritisch gerade in Diskursen, die technizistisch argumentierend die digitale Qualität der Filme ausstellen, nach den zugrunde liegenden Mustern, verwendeten Bildern und wahrnehmungstheoretischen Implikationen fragen. Daran schließt sich die Untersuchung an, wie sich das Label digital im marktorientierten Kontext in mediale Phänomene übersetzt.

Ein solcher kultur- und medienkritischer Ansatz strukturiert mit etwas anderer Begrifflichkeit als bei Winkler auch die Ausgangsüberlegungen des Sammelbandes Digitalität und Kino zur Mediengeschichte (Segeberg 2012a). Der Band stellt die Frage in den Mittelpunkt, ob es möglich wäre, die Medialität der Filmgeschichte jenseits einer immer wieder unterstellten Dichotomie aus

33 Im Folgenden thematisiert Winkler das Problem der wissenschaftlichen Erkenntnis selbst. Besonders interessant ist hier, dass er diese Überlegungen mit (inner)disziplinären und fachpolitischen Entwicklungen verbindet. So diskutiert er die Vorzüge von technikzentrierten Ansätzen mit Blick auf das herkömmliche Vorurteil von harten (rationalen) Naturwissenschaften vs. weichen Humanwissenschaften und ihren Gegenstandsbereichen, die nicht so klar bestimmbar seien wie in den Naturwissenschaften. 
Analog und Digital zu bestimmen: „Welchen Beitrag zur Beschreibung [und Konstitution einer Vorstellung, FH] des Digitalen leisten die eher generellen [sich historisch in die Mediengeschichte einordnenden, FH] Diskurstypen zum Digitalen" (Segeberg 2012b:13, Hervorh. FH)? Anstatt immer auf die Differenzen hinzuweisen, ${ }^{34}$ solle man vielmehr den medienhistorischen Kontext und die Genese digitaler Technologien deutlicher verorten und kontextualisieren.

Segeberg benennt - ähnlich wie Winkler - zwei Diskursmodelle der Mediengeschichte, zwischen deren Polen sich die Einschätzung der Rolle von Film beziehungsweise Kino bewegt:

- Das bellizistische Modell beschreibt die Geschichte der Medien als einen Krieg unter Medien, in dem ein Medium das andere dadurch so gut wie auslöscht, dass es dessen Leistungen so komplett wie irgend möglich in sich aufnimmt, weiterentwickelt und überbietet (Segeberg 2012b: 15).

- Das medienökologische Modell nimmt dagegen an, dass Medien einander nicht auslöschen, sondern sich in der Regel im Verbund mit anderen Medien - und das hieße koevolutiv - entwickeln. In diesem Erklärungsmodell ${ }^{35}$ wechseln daher nicht die Medien als ganze, sondern die Leit- oder Führungsrollen. Dabei überleben die zu einem Zeitpunkt nicht dominanten Medien nicht selten in "mit Artenschutz versehenen medienökologischen Nischen" und können unter anderen zeitlich-situativen Bedingungen erstaunlich erfolgreich revitalisiert werden (Segeberg 2012b: 15).

Im Horizont eines solchen medienökologischen Modells, das von einer koevolutiven Dynamik ausgeht, sind meines Erachtens viel zitierte Ansätze wie vor allem der der Remediation von Bolter/Grusin (vgl. ausführlicher hierzu $\rightarrow$ Kap. 4) zu sehen, die 1999 auf die sich verdichtende Multimedialität von Phänomenen reagierten und suchten, diese konzeptuell und begrifflich fassbar zu machen. Flückiger konstatiert mit Blick auf die von Bolter/ Grusin fokussierten Phänomene Prinzipien des ständigen Konfigurierens und Umkonfigurierens von Erscheinungsformen und Inhalten in bestimmten kulturellen Rahmungen. Diese (ständigen) Konfigurationsprozesse sind als Netzwerk zu denken, bei dem es keinen Kern, keine irreduzible Essenz mehr gibt, die eindeutig auf ein Ursprungs- beziehungsweise Originalmedium verweisen (Flückiger 2008: 400). ${ }^{36}$

34 Vgl. hierzu auch den Begriff von Belton (2003) einer "digitalen Scheinrevolution“.

35 Dieses wird vor allem von dem Medienökologen Michael Giesecke favorisiert.

36 Nach Fossatis Analyse (2009) archivarischer und restauratorischer Praktiken sind dies Formen der praktischen Umsetzung des Remediation-Modells (2009:137 f.): Mithilfe eines neuen Mediums (digitale Domäne) wird ein älteres (fotochemischer Abbildungsprozess) simuliert und in der Rezeption (= restaurierter Film) möglichst ununterscheidbar gemacht (2009: 140, 218). 
Methodisch gewendet heißt dies nach Thorburn/H. Jenkins in Rethinking Media Change (2004), dass man eher eine komparative Herangehensweise an mediale Phänomene und ihre Aktualisierungen von zeitlich früheren Phänomenen wählt und keine kompetitiv betriebene Suche nach einer Medienspezifik mehr vornimmt ${ }^{37}$ - dies helfe auch, einseitig utopische wie dystopische Einschätzungen eines Medienwandels zu vermeiden (Thorburn/H. Jenkins 2004: 1). Vor diesem Hintergrund solle man alt und neu weniger in einem kompetitiven Verhältnis denken als vielmehr als kollaboratives Netzwerk im Kontext einer sich im ständigen Wandel befindenden medialen und kulturellen Umgebung (Thorburn/H. Jenkins 2004: 3). ${ }^{38}$ So plädieren Thorburn/H. Jenkins auch für ein Gedankenmodell der Evolution statt der bellizistischen Revolution. ${ }^{39}$ In diesem Horizont profilieren sie gleichermaßen methodologisch die Notwendigkeit einer pragmatischen Herangehensweise, die eine Kontextualisierung der jeweiligen Bedingungen von technologischem Wandel und Entwicklung bestimmt. ${ }^{40}$

Als kurzes Zwischenfazit sei darauf verwiesen, dass hier kumulativ verschiedene Ansätze vorgestellt wurden, die - in je unterschiedlicher Gewichtung - das Verhältnis von Technologieentwicklung, erlebbaren Phänomenen und Dynamiken soziokultureller Praktiken zusammendenken: Fossati (2009) mit Blick auf das Modell der SCOT-Theorie mit einem Schwerpunkt auf den beteiligten Akteuren in dem Feld; Winkler (2000), historisch zum Jahrtausendwechsel eingebunden in eine disziplinäre Methodendebatte der deutschsprachigen Medienwissenschaft, versucht zwei Modelle der Mediengeschichtsschreibung in einer zyklischen Struktur miteinander zu vermitteln und profiliert gleichzeitig einen kulturkritischen Ansatz auch bei den technizistisch ausgerichteten Diskursen. Dieser findet seine Wiederaufnahme bei Segeberg (2012b). Allerdings richtet Segeberg den Fokus auf die

37 „Medium-specific approaches risk simplifying technological change to a zero-sum game in which one medium gains at the expense of the rivals" (Thorburn/H. Jenkins 2004: 3).

38 "[T]o focus exclusively on competition or tension between media systems may impair our recognition of significant hybrid or collaborative forms that often emerge during times of media transition" (Thorburn/H. Jenkins 2004: 3).

39 Nach Thorburn/H. Jenkins (2004) sind entscheidende Prinzipien historiografischer Dynamik, damit sich überhaupt Kategorien wie alt und neu herauskristallisieren können, zum einen die Institutionalisierung, zum anderen die Standardisierung - beides kulturelle, gesellschaftliche und ökonomische Prozesse, gepaart mit Technikentwicklung.

40 „In our current technological transition, there is an urgent need for a pragmatic, historically informed perspective that maps a sensible middle ground between the euphoria and the panic surrounding new media, a perspective that aims to understand the place of economic, political, legal, social and cultural institutions in mediating and partly shaping technological change" (Thorburn/H. Jenkins 2004: 2). 
diskursiven und zeitlichen Verhältnisse medialer Phänomene untereinander. Er rekurriert damit auf zentrale Positionen von Bolter/Grusin (1999) sowie Thorburn/H. Jenkins (2004), die angesichts des Multimedialen versuchen, die geschichtliche Entwicklungsdynamik beschreibbar zu machen.

Aus dieser Auffächerung der Perspektiven ergeben sich für die vorliegende Studie folgende Konsequenzen:

- Angesichts des Phänomens von digitalisierten Filmen wird von der Dynamik eines koevolutiven, vielschichtigen Netzwerkmodells ausgegangen. Dies findet sich methodologisch darin umgesetzt, dass die Analysekapitel mit ihren jeweiligen Schwerpunkten zu einem spezifischen diskurskritischen und wirkungsästhetischen Topos als Cluster (Spielmann 1998) verstanden werden, in denen mehrere historiografisch wirksame Schichten als Wahrnehmungsoptionen koexistierend simultan aktiv werden.

- In Anlehnung an die oben diskutierten Überlegungen erhält der Begriff der Transition eine zentrale Funktion - in bewusst mehrdeutigem Sinn. Er bezeichnet zum einen die zeitliche Dynamik als Fluss, in dem die Vergangenheit mit der Gegenwart interagiert und die Zukunft in der Gegenwart schon projektiv enthalten ist. ${ }^{41}$ Zum anderen wird Transition als ständige Bewegung gedacht, als ständiger Prozess der Aushandlung. Darüber hinaus hat der Begriff der Transition das Potenzial, der immanenten Dynamik des zyklischen Verhältnisses von menschlichen Bedürfnissen und Technologieentwicklungen Rechnung zu tragen.

\section{Digitalisierung, Gesellschaft, Ästhetik und Geschichte}

In diesem zweiten Abschnitt des $\rightarrow$ Kap. 2 - der erste widmete sich Begriffen und Konzepten ${ }^{42}$ - wird die bereits angesprochene kulturkritische Perspektive mit der Darstellung unterschiedlicher Ansätze in ihren Facetten dimensioniert und zugleich mit Blick auf den Gegenstandsbereich der vorliegenden Studie präzisiert. Zunächst werden bereits in Teil I angesprochene technologische Voraussetzungen in ihren Konsequenzen für Welt- und Wahrnehmungskonzepte erläutert. Daran anschließend wird der Rückgriff auf Fredric Jameson

41 Dies erinnert an das zeitphilosophische Konzept der "gedächtnishaften, erlebten Zeit“, der Dauer/durée nach Henri Bergson (1991/1896), die vom Raum entbunden ist. Vgl. in geschichtstheoretischer Perspektive die Ausführungen zu Konzepten von Koselleck in $\rightarrow$ Kap. 1.

42 Vgl. den zweiteiligen Aufbau dieses $\rightarrow$ Kap. 2 in Begriffe und Konzepte und Digitalisierung, Gesellschaft, Ästhetik und Geschichte. Vgl. überblicksartig die Erläuterung zu diesem Gliederungsprinzip in der Einleitung $\rightarrow$ Kap. 1. 
und seine Kulturdiagnose für ,postmoderne', ,spätkapitalistische' Gesellschaftsstrukturen seit den 1980er Jahren vorgenommen. Wichtig wird hier insbesondere die Vermittlung Jamesons sein, der ästhetische Prinzipien und Vorstellungen von Historizität aufeinander bezieht. Mit diesem Rekurs wagt meine Argumentation historisch einen Schritt zurück: Zum einen ermöglicht dies das Aufzeigen der historischen Linie von memo- und kulturpolitischen Problemkomplexen und Diskursen, die mit Computertechnologien nicht erst in den 2oooern Jahren aufgekommen sind. Mit der konkreten Verbindung von Jamesons Kulturdiagnose und der Colorization-Debatte der 198oer Jahre können grundlegende Problemstellungen und ästhetische Phänomene in ihrer Entwicklung aufgezeigt werden, die zu Beginn der 2oooer Jahre als digitale Tools verbreiteter zum Einsatz kamen, noch prominenter zutage treten. So schließt das Kapitel mit dem Fallbeispiel der deutschen TV-Serie WeltenBRAND aus dem Jahre 2012, welches das zu beschreitende Spannungsfeld zwischen medientechnologischen und memo- wie kulturpolitischen Diskursen einerseits und Modellierungen der medien- und wirkungsästhetischen Dimensionen anderseits am Gegenstand entfaltet.

\subsection{Welt- und Wahrnehmungskonzepte im Zeichen des Digitalen. Quantisierung, Vergleichbarkeit und Effizienz}

Als besonders grundlegend für die Verbindung von Funktionsweisen digitaler Technologien und sozialer, gesellschaftlicher und gedanklicher Konfigurationen gilt eine Studie von Mark J. P. Wolf. Wolf hat in Abstracting Reality. Art, Communication, and Cognition in the Digital Age (2000) gezeigt, wie sich Prinzipien digitaler Ordnungen und Denkmuster in die Kultur einschreiben und in Perzeptionsformen übersetzen können. Wolf untersucht die Interaktion zwischen digitaler Aufzeichnung, Wahrnehmung und Kognition. Seine These lautet, dass die Künste und Medien, die ihre Abbildungen durch einen technischen Aufzeichnungsprozess herstellen (wie die Fotografie, der Film, die Tonaufnahme), sich in einem Abstraktions- und Dematerialisierungsprozess zu einer quantisierten, zunehmend mediatisierten Beziehung zur Wirklichkeit hinbewegen. Mit digitalen Technologien und einhergehenden Denkmustern findet vor diesem Hintergrund eine Verlagerung vom perceptual zum conceptual statt. Eine Ebene der Abstraktion wird eingezogen, denn „analog and digital represent concepts" (Wolf 2000: ix). Dieser Abstraktionsprozess hat Folgen für die Vorstellung von historischer Dynamik: „It extends beyond a form of design into a way of thinking, an attitude towards the world and the future" (Wolf 2000: xi). 
Wolfs Überlegungen lassen sich meines Erachtens im Ansatz mit Grundgedanken des Philosophen Gilles Deleuze vergleichen, wenn es um das produktive Verhältnis der Modellierung von Denken, medialen Formen und Raumzeitlichkeit geht: Ist für Deleuze das (moderne) Filmbild als Zeit-Bild (bes. 1997b/1985), das heißt als eine Gegenverwirklichung modernen Denkens zu verstehen, könnte man weitergehend formulieren, dass Wolf etwas Ähnliches für digitale Technologien vorschlägt: Die raumzeitliche Ordnung digitaler Daten - diskret, diskontinuierlich und vor allem quantisierterscheint als eine Gegenverwirklichung aktuell geläufiger Weltkonzeptionen und Geschichtsvorstellungen. Wolf beschreibt die Dynamiken zwischen soziokulturellen und technologischen Entwicklungen als eine komplexe prozessuale Interaktion (Flückiger 2008: 38). Im Horizont des zentralen Prinzips von Quantisierung stellen hierbei die Diskretheit von Werten sowie das Verfahren der (abstrakten), Repräsentation' Schlüsselkonzepte dar (Wolf 2000: $\mathrm{x}$ ). Diese forcieren die kulturelle, diskursive und erkenntnistheoretische Stellung von mathematisch messbarer Welterfassung (hier verweist Wolf auf den von Donna Haraway [1985] benannten „Messwahn“). Ähnlich wie Schröter 2004 konstatiert Wolf hierbei, dass analog und digital zu ideologisch aufgeladenen Synonymen geraten:

[A]nother difference between analog and digital is their connotative meaning in popular usage. In common parlance, ,digital' has come to represent the modern, state-of-the-art technology, while ,analog refers to an older, outmoded and outdated form. [...] The term, digital' is also often associated with a high degree of quality, even though the term refers to a technology and not a specific application of it (Wolf 2000: $\mathrm{x}$ ).

Vor diesem Hintergrund sind auch digitale Bilder und ihre soziokulturelle Bedeutung einzuordnen. Nach Wolf haben gerade diese Einzug in das alltägliche Leben gehalten, ihre Logik prägt damit einen bestimmten Typus von Gesellschaft. ${ }^{43}$ Die wachsende Informationsgesellschaft, die zugleich Bedingung

43 In diesem Horizont hat in jüngerer Zeit auch das Schlagwort Postdigitalität an Popularität gewonnen, was einen weiteren Schritt in der kulturwissenschaftlichen Beschäftigung mit digitalen Phänomenen bedeutet. In dem Begriff äußert sich die Vorstellung, dass es weniger gilt, die „disruptiven Folgen der Digitalisierung“ (Settembrini di Novetre 2012: 2) zu benennen, als vielmehr zu konzeptualisieren, dass die Digitalisierung den Alltag erfasst hat und damit nicht mehr der Sphäre der Virtualität und Dematerialisierung zuzuordnen ist: „Postdigital heißt gerade nicht, dass digitale Technologien und digitale Medien keine Rolle mehr spielen. Genau das Gegenteil ist der Fall: Die tiefe und nachhaltige Durchsetzung der Digitalisierung ist eine notwendige Bedingung für den postdigitalen Zustand“ (Köhler, zit. n. Settembrini di Novetre 2012: 2). Der Blogger Settembrini weiter: „Oder anders gesagt: post- meint nicht anti-, so wie die Postmoderne ja auch keine 
wie Resultat der Entwicklung ist, bildet hier den „frame of mind“ und liefert „ways of thinking“; diese Denkmuster lassen wiederum digitale Technologien begehrenswert und notwendig für das alltägliche Leben erscheinen (Wolf 200o: xi). Quantisierung strukturiert so vier grundlegende Kategorien unserer Welterfahrung und -verständigung, indem diese in diskrete, abstrakte Werte eingeteilt werden: Zeit, Raum, Wert und Information. Diese standardisierten abstrakten Einteilungen bilden die Grundlage und Voraussetzung für die (stetige) Vereinfachung von Austausch, Kommunikation und Handel (Wolf 2000: 5$)^{44}$

Für unseren Zusammenhang - gerade auch mit Blick auf die Beschäftigung mit ästhetischen Bildphänomenen und deren Zirkulation - interessiert besonders das Austauschverhältnis von Mechanismen der soziokulturellen, vor allem ökonomischen Wertbestimmungen und digitalen Technologien. Die Konsequenzen des Prinzips von Quantisierung werden von Wolf zunächst wie folgt beschrieben:

\begin{abstract}
Although value itself is always in flux, just as the values of currencies fluctuate, it is still quantized into numerically expressible amounts. The need to quantify value came about with trade and the need to insure that an equitable exchange had been made, since trade was an impetus for the development of mathematics itself (Wolf 2000: 14).
\end{abstract}

Vor diesem Hintergrund sei an Holert (2002) erinnert, der den Zusammenhang von neoliberalen, globalisierten Weltkonzeptionen, Finanzsystemen und digitalen Technologien hervorgehoben und damit die (ideologisch-ikonografische) Verwebung von marktwirtschaftlichen Prinzipien und Zirkulationen mit der Vermessung von Informationen und Bildphänomenen beschrieben hat. Laut Wolf haben die damit einhergehenden Dematerialisierungs- und Abstraktionsprozesse, denen Informationen und Phänomene unterworfen werden, zum einen De- und Re-Kontextualisierungen ebenjener zur Folge; zum anderen etabliert sich damit (paradoxerweise) ein mathematischer, an Zahlen orientierter Begriff von Objektivität (Wolf 2000: 21), der etwa in ökonomischen Kontexten als Grundlage der Wertbestimmung funktionalisiert werden kann.

Gegenmoderne darstellt, sondern auf den Errungenschaften der Moderne gewissermaßen aufsetzt.“ (Settembrini di Novetre 2012: 1). In diesem Horizont seien auch „sich überlappende Retro- und Nostalgiewellen“ zu sehen.

44 Die Einführung einer Geldwährung - Münzen als abstrakter Gegenwert für Naturalien ist hier eines der zentralen Beispiele Wolfs. 
Schon Wolf warnt hier - und dieses Problem wird sich in meiner Auseinandersetzung mit Filmen und Filmbildern als ästhetischen Phänomenen noch zuspitzen -, dass die Orientierung an diskreten, kleinen Einteilungen Gefahren birgt, da sie reduktionistisch wirkt ${ }^{45}$ und die menschliche Existenz in binäre Ordnungsprinzipien presst. Das Denken in der Logik des Digitalen („digital-style“) gehorcht einem utilitaristischen Effizienzgedanken, was zu stereotypen Kategorisierungen und Vereinfachungen führt (Wolf 2000: 22). In Konsequenz leistet dies Denkansätzen Vorschub, bei denen es darum geht, die eine, nämlich „one best solution“ zu finden, um ein Maximum an messbarer Effizienz und ökonomischer Verwertbarkeit zu erreichen. Und damit verbindet sich auch die Vorstellung von (teleologischem) Fortschritt, der wiederum messbar bleiben muss (Wolf 2000: 23).

Vor diesem Hintergrund der Prinzipien der Abstraktion, Dematerialisierung und Repräsentation eröffnen sich mit Blick auf ästhetische Phänomene und den Kunstbegriff, nicht zuletzt im Kontext von Film, nachhaltig Spannungsfelder, da - in Erinnerung an Benjamin und die Aspekte der technischen Reproduzierbarkeit - Begriffe von Authentizität (unter anderem gebunden an ein Konzept von klar verortbarer Urheberschaft) prekär werden. Hauptreferenz an dieser Stelle, auf die sich nicht nur Wolf (2000), sondern auch Rodowick (2007), Flückiger (2008), Fossati (2009) beziehen, sind die symboltheoretischen Überlegungen des analytischen (Kunst-)Philosophen Nelson Goodman. Dessen berühmt gewordene Unterscheidung zwischen autografischen und allografischen Künsten (Goodman 1997/1968: 122 ff.) weist im Kontext des Films in Hinblick auf Konzepte von Original und Kopie auf schwer zu klärende Fragen hin. Film hat für Rodowick einen hybriden und schwierigen Status (Rodowick 2007: 14-15): Ist für die Identität von autografischen Kunstwerken nach Goodman entscheidend, dass sie an ihre physische Identifikation gebunden sind und damit auch an die Idee eines physischen Erzeugnisses des Künstlers (etwa bei einem Gemälde), so sind allografische Künste zweistufig, da der Künstler eine Notation entwirft, auf deren Grundlage das Kunstwerk in verschiedenen Realisationen von ihm emanzipiert aufgeführt werden kann. Zentrales Beispiel ist die Musik, in der die Komposition als individueller, kreativer Akt zeitlich und räumlich getrennt sein kann vom performativen Akt des Kunstwerks - auf der Grundlage des Notationssystems zum Beispiel einer Partitur. Bereits der analoge Film mit seinem Prinzip der technischen Reproduzierbarkeit verortet sich zwischen den beiden Kategorien, da

45 "Quantization is inherently reductionist; by its very nature it seeks to find or create divisions, its representations are discrete rather than continuous“ (Wolf 2000: 21). 
er offensichtlich zwei- oder gar mehrstufig funktioniert. Doch wo ist beim Film - äquivalent zur Unterscheidung zwischen Komposition/Notation und Performance - die Grenze zu ziehen (Rodowick 2007: 15)? Was ist bei ihm die Vorlage beziehungsweise die Notation, was die Aufführung?

Dies verkompliziert sich nun angesichts der Wandlung der Form der Reproduzierbarkeit:

The digital image extends these problems [...]. Computer generated images are not autographic for two reasons: as ,synthetic images they cannot be considered the physical act of the author's hand, nor do they result in an end product. Indeed one of the great creative powers of digital images is their lack of closure, a quality Philip Rosen has characterized as ,practically infinite manipulability' they are easily reworked, reappropriated, and recontextualized (Rodowick 2007: 15).

Mit Rekurs auf Stanley Cavell bezeichnet Rodowick dies auch als die Automatismen der digitalen Künste. In dieser Hinsicht stellen sie sich als antagonistisch zu autografischen Kunstwerken dar. Allerdings ist es in der digitalen Domäne möglich, eine augenscheinlich fast identische Kopie des originalen Kunstwerks herzustellen. Auf der technischen Ebene könnte der zugrunde liegende Algorithmus als Notation des Bildes verstanden werden, seine Sichtbarmachung in Gestalt eines Filmbildes als performativer Akt, als „Aufführung“ (Rodowick 2007: 15-16).

Dies kann mit weiteren grundsätzlichen Überlegungen von Goodman (1997) zusammengebracht und methodologisch gewendet werden: Eigenschaften und Symbolhorizonte, die Kunst und damit auch den digitalen Film ausmachen, sind nicht unabhängig von den jeweiligen Rezeptionskontexten zu sehen. So bedeutet das Wahrnehmungscluster digitalisierter Film zu verschiedenen Zeiten verschiedene Konfigurationen von Sinn (vgl. hierzu zum Prinzip der Relationen $\rightarrow$ Kap. 7). Daher untersuche ich digitalisierte Filme als (Symbol-)Systeme dahingehend, inwiefern Digitalisate und damit implizit auch die vermittelten Geschichtsbildmodellierungen je nach Kontext und Bezugsrahmen als (auch außermedial) bedeutsam und richtig empfunden werden können. ${ }^{46}$

46 Vor diesem Hintergrund wird auch in $\rightarrow$ Kap. 7 nach den digitalen Konfigurationen gefragt, in denen über verschiedene Symbolsysteme und deren innere Relationen Versionen von Geschichts- und damit auch Weltmodellierungen vermittelt werden. Auch wenn dies von der vorliegenden Studie im phänomenologisch imprägnierten Ansatz nicht grundlegend systematisch übernommen wird, so könnte man die Funktionsweisen eines kognitiven ,Weltenbaus' auf der Grundlage von Symbolen nach einer digitalen Logik in einer solchen Konfiguration mit Nelson Goodman (1990/1978) beschreibbar 
All die hier vorgestellten Überlegungen eröffnen darüber hinaus noch viel breitere Spannungsfelder - gerade im Zusammenhang mit Denkmustern, die von Logiken der Quantisierung und der Vermessung geprägt sind. Die Friktionen werden besonders offensichtlich, wenn man ebendiese Logiken auf Kulturgüter und Phänomene der ästhetischen Wahrnehmung bezieht (Heller/ Flückiger 2010).

In der Praxis haben Versuche, Filmdigitalisierungsprozesse im Dienste von Logiken der Effizienz zu automatisieren, zahlreiche offene methodische Fragen und ethische Herausforderungen zutage treten lassen (u. a. Fossati 2009; Heller/Flückiger 2010; Flückiger 2018). Exemplarisch wird dies deutlich bei der Debatte um den ethisch vertretbaren, bis heute nicht standardisierten Umgang mit den ästhetischen Eigenschaften von historischen Filmbildern, wenn sie automatisiert in die digitale Domäne überführt und damit in ihrer digitalen Qualität potenziell in jeglicher Hinsicht modellierbar werden. Diese Programmierbarkeit beziehungsweise der automatisierte Umgang mit den konkreten Phänomenen steht in vielen Fällen im Kontext einer Einschätzung des (unterhaltenden ästhetischen) Werts für die heutigen Medienumgebung.

Ähnliche Probleme ergeben sich aber auch schon vorgelagert, wenn es um die Selektion geht, was überhaupt wert ist, in die digitale Domäne überführt zu werden. Hier kommen weiterhin die (Un-)Möglichkeiten einer messbaren Wertbestimmung von Filmen zum Tragen ${ }^{47}$ (vgl. hierzu die ausführlicheren Erläuterungen zu Werterelationen des Ästhetischen im Kontext des Films in $\rightarrow$ Kap. 6 und $\rightarrow$ Kap. 7). Wichtig ist Wolfs Hinweis an späterer Stelle, dass Vermessung oft eine Wahrnehmungsformierung und -haltung des Vergleichens bedingt (Wolf 200o: 22; vgl. hierzu auch $\rightarrow$ Kap. 7).

Ein solche Konfiguration des Vergleichens geht auf den hier zum Abschluss profilierten zentralen Diskursstrang im Zeichen digitaler Denkmuster zurück: Als Flusser die Krise der Linearität (1992) anhand der Hypothese untersuchte, dass unser Denken, Fühlen, Wünschen, Handeln, Wahrnehmen und sogar Vorstellen - wie wir die Welt und uns selbst erfahren - durch die Struktur von Codes geformt wird, da hob er besonders das Prinzip hervor, das aus der Möglichkeit des Random Access digitaler Daten entspringt. Gerade hier artikuliert sich die Loslösung von linearen Denkmustern und Repräsentationsmustern. Random Access ermöglicht die Bildung von netzwerkartigen Strukturen: Statt der Logik von Buchstaben in Zeilen zu folgen, zerbröckelt die mathematische

machen: Komposition/Dekomposition, Gewichtung, Ordnen, Tilgung und Ergänzung, Deformation.

47 Zur Erinnerung Wolfs grundsätzlicher Befund: „Measurability and exchange value have become too important in the estimation of worth“ (Wolf 2000: 23). 
Logik Oberflächen des Bildes in Punkte und Intervalle (Flusser 1992: 22). Es sei ein „Aufstand der Zahlen gegen die Buchstaben“, der das Denken in Punkte „zerklaubt“ (Flusser 1992: 23).

Manovich (2001: u. a. 244-285) hat diesen Ansatz mit den Konzepten der Datenbank weiter ausgebaut und vor allem auch die dadurch entstehenden Navigationsräume beschrieben. Distelmeyer (2012) greift seinerseits den sich darum rankenden Diskursstrang auf und bezieht das Prinzip der Verräumlichung - auch im Vermittlungsmodus einer interaktiven, (nach) vollziehenden Kartografie - auf die konkreten digitalen Erscheinungsbedingungen von Film in entsprechenden Dispositiven (Distelmeyer 2012: $170 \mathrm{ff}$.). So verortet er die Vermittlung der Idee von digital in dem Prinzip der Verräumlichung und in dessen oft ostentativem Ausstellen seitens der medialen Anordnungen (zum Beispiel DVD oder Blu-Ray-Disc, Distelmeyer 2012: 172). Die jeweiligen (räumlichen) Konfigurationen sind - unter Bezug auf Wolfs bereits zitierten Hinweis (Wolf 2000: 22) - auf die Art und Weise hin zu prüfen, wie sie Modi des Vergleichs von Phänomenen zum Zwecke einer ökonomisch umsetzbaren Wertzuschreibung gestalten. Solche medialen Vermittlungsformen der Vorstellungen und Konzeptionen von ,digitaler Logik und ,digitaler Welterfahrung ' werden wiederkehrend in den Analysekapiteln Gegenstand sein (vgl. insbesondere $\rightarrow$ Kap. 7 zu digitalen Konfigurationen des Vergleichens; aber auch $\rightarrow$ Kap. 5 zur historiografisch-performativen Dimension von verräumlichten Vermittlungsformen von Film).

\subsection{Postmoderne Strukturen und der Verlust des Historischen}

Fredric Jameson hat bereits in den 198oer Jahren Gesellschaftsstrukturen als "spätkapitalistische" in den Blick genommen und dabei die Kategorie Postmoderne als "kulturelle Dominante" benannt. ${ }^{48}$ Unter diesen Vorzeichen sei ästhetische Produktion zunehmend zu einer Ware, zu einem ökonomisch verwertbaren Gebrauchsgegenstand geworden (Jameson 1991: 4).49 Vor diesem Hintergrund beschreibt Jameson insbesondere die imaginäre und transzendentale Dimension, die in einem solchen gesellschaftlichen Umfeld digitalen Technologieentwicklungen zugeschrieben wird. Diese artikulieren

48 "[I]t seems to me essential to grasp postmodernism not as a style but rather as a cultural dominant: a conception which allows for the presence and coexistence of a range of very different, yet subordinate features" (Jameson 1991: 4).

49 Einige Kapitel des 1991 erschienenen Buches von Jameson wurden bereits einzeln in den 1980er Jahren publiziert. 
sich in Form von "[ $[$ ] antasies about the salvational nature of high technology, from chips to robots" (Jameson 1991: 46).

Jamesons Überlegungen weisen im kulturkritischen Ansatz Gemeinsamkeiten mit bereits diskutierten Positionen auf. In seiner Argumentation zeigen sich ähnliche Schlüsselprinzipien der Haltung zu Geschichte und Vergangenheit: Diskontinuität, Dematerialisierung, Abstraktion von Werten und Deterritorialität. Diese Prinzipien werden in Jamesons Perspektive immer wieder mit Strukturen einer kapitalistischen Gesellschaftsordnung und deren Interessen zusammengebracht.

Diese Kombination zeitigt gravierende Folgen für das Geschichtsverständnis: „For I take it axiomatic that ,modernist history“ is the first casualty and mysterious absence of the postmodernism period" (Jameson 1991: xi). Dadurch, dass ästhetische Produktionen in die Warenproduktion integriert werden (Jameson 1991: 4), erfolge ein Verlust von Historizität (Jameson 1991: $\mathrm{x}$ ). Die Konsequenz sei eine neue Oberflächlichkeit und damit fehlende Tiefe, die in einer neuen Kultur des (Ab-)Bildes oder des Simulakrums eine Verlängerung finde, welches in neuer Funktion seinen eigentlichen Wurzeln entrissen werde. Dies bedeute

[a] consequent weakening of historicity, both in our relationship to public History and in the new forms of our private temporality [...]; the deep constitutive relationships of all this [relate/go back/refer, FH] to a whole new technology, which is itself a figure for a whole new economic work system (Jameson 1991: 6).

Jameson beschreibt das weite Netzwerk des Zusammenwirkens von neuen Technologien und einer marktwirtschaftlichen ökonomischen Gesellschaftsordnung, die in Richtung eines globalisierten (Kapital-)Raums strebt, in dem es grundsätzlich - ob im privaten oder im kollektiven, öffentlichen Geschichtsverständnis - zu einem Verlust von historischer Identität kommt. Die Stabilität des historischen Referenten wird dadurch unterminiert. Dies zeitigt seinerseits kunstpolitische Konsequenzen: „[O]ne fundamental feature of all the postmodernisms enumerated above: namely, the effacement in them of the older (essentially high-modernist) frontier between high culture and so-called mass or commercial culture" (Jameson 1991:2). Das Verschwimmen der Grenze zwischen elitärer Hochkultur und massenmedialer Kultur finde in einer bestimmten Ästhetik, in der Form des Recyclings und Zitierens, seinen Ausdruck. Dies verbinde sich mit den Prinzipien der Dekontextualisierung und kulminiere im ästhetischen Prinzip des Pastiches (Jameson 1991: 16). ${ }^{50}$ 
Dadurch, dass historische Identität prekär werde, könne man sich hergebrachter Motive und Symbole bedienen, die in der Re-Aktualisierung und der Re-Kontextualisierung des (Gebrauchs-)Zusammenhangs imitierend neu perspektiviert würden, wobei der Warenwert in der gegenwärtigen Wahrnehmung eine dominierende Rolle spiele. Die Erinnerung an den Gebrauchskontext und -wert, den das Zitierte ursprünglich gehabt habe, trete zurück: "The culture of the simulacrum comes to life in a society where exchange value has been generalized to the point at which the very memory of use value is effaced" (Jameson 1991: 18). ${ }^{51}$ Die Vergangenheit, das, was als ,historisch' wahrgenommen wird, ist nur noch eine weite Sammlung von (Ab-)Bildern, die auf nichts anderes mehr als auf sich selbst verweisen - in einer räumlichen, synchron organisierten Anordnung (,the new spatial logic“, Jameson 1991:18 f.).

Nach diesen Überlegungen Jamesons wird im methodischen Horizont meiner Studie nahegelegt, Geschichtlichkeit als Effekt eines konkreten medialen Ausdrucks in den Blick zu nehmen - unter der dominanten Maßgabe des zugeschriebenen Marktwerts. ${ }^{52}$ Mit Rekurs auf Barthes spricht Jameson hier vom Vergangenheitseffekt, dem Effekt einer pastness (Jameson 1991: 19).

Bezogen auf den Gegenstandsbereich meiner Untersuchung lassen sich so Jamesons Überlegungen vielschichtig aufgreifen. Die Destabilisierung der historischen Referenz, der ursprünglichen Zusammenhänge und die pasticheartigen Wiederaufnahmen von historischem Material sind in unterschiedlichsten (digitalen) Dispositiven zu beobachten, wie sie zum Beispiel Broeren (2009) hinsichtlich der Funktion und Bedeutung von YouTube reflektiert. Michael Loebenstein benennt 2009 in einem Vortrag mit Blick auf die unkuratierte Onlinestellung von historischem Material den Prozess der Dekontextualisierung als „Amazonisierung im digitalen Marketplace“ (Loebenstein 2009; Cherchi Usai et al. 2008). Alexander Horwath, ehemaliger Leiter des einflussreichen Österreichischen Filmmuseums, geht noch einen Schritt weiter. Schon 2005 machte er eine "neo-liberale Rhetorik“ aus, die den Begriff digital diskursiv überforme; dieser interagiere mit Ausdrücken wie User-driven, Content Management, Content on Demand, die bedeuteten, dass

such mimicry, without any of parody's ulterior motives, amputated of the satiric impulse“ (Jameson 1991: 17).

$5^{1}$ Zum Begriff des Simulakrums rekurriert Jameson auf Platons entsprechendes Konzept: „[T] he identical copy for which no original has ever existed“ (Jameson 1991: 18).

52 Die Wahrnehmungskonfiguration in diesen raumzeitlichen Strukturen beschreibt Jameson als "nostalgia modes of reception“ (Jameson 1991: 19). Als ästhetische Zeugnisse hierfür zitiert er Filminhalte. Die Befunde arbeitet Jameson vor allem anhand der Analyse der Storyebene etwa der Filme Something Wild (1986) und Blue Velvet (1986) heraus. 
allein die Filminhalte (eben nicht Film als historische Praxis oder materielles Artefakt) sich in einem Free Flow befänden, wenn sie etwa online, ohne kuratorische Betreuung oder Rahmung zur Verfügung stünden (Horwath 2005: 7 f.). Die Nachfrage bestimme den Markt und damit dann zusehends das Angebot der Filmgeschichte. Dabei gerieten - und dies ist auch der Untersuchungsbereich der vorliegenden Studie - die Bedeutung und Referenzfunktion des historischen Materials nachhaltig in Abhängigkeit von seinen augenblicklichen Präsentations- und Distributionsformen.

\subsection{Historisches Fallbeispiel: Memopolitik im Zeichen von Computertechnologien und Medienwechseln. Die Colorization- Debatte der 198oer Jahre}

„The DVD era is resurrecting the great colorization debate of the $198 \mathrm{os}^{\text {“ }}$

(Associated Press 2004).

Einen besonderen historischen Fall, bei dem die technologische Entwicklung, ökonomische Interessen, neue Medienumgebungen und ästhetische Formen in ein Spannungsfeld gerieten, stellte in den 198oern die Praxis der sogenannten Colorization oder Color Conversion dar (Klawans 1990: 159). Colorization ${ }^{53}$ für den amerikanischen Fernseh- und für den Videomarkt war eine historische Praxis, bei der audiovisuelles Erbe als ökonomischer Gebrauchswert ästhetisch verändert und ,marktgerecht' aktualisiert wurde: „Colorization is the computerized process of adding color [sic!] to black and white films. It is employed to re-invent a market for films" (Acland 1990: 12).

Diese Praxis löste eine breite, öffentlich geführte Debatte in den USA aus (Klawans 1990; Acland 199o; Bohn 2013b: bes. 269 sowie Op den Kamp 2015 und 2018 mit Schwerpunkt auf den juristischen Bedingungen). Sowohl die dokumentierten Beweggründe der ausführenden Firmen und Sender (vor allem unter Ted Turner) sowie die (publizistischen) Reaktionen bezeugen die Bandbreite einer Diskussion, die Problemstellungen und Dimensionen digitaler, marktorientierter Eingriffe in historische Filme und deren Vermarktung bis heute aufwerfen. Was an dem Beispiel sichtbar wird, ist das Zusammenwirken von technologischen und medialen Bedingungen, die mit einem Medienwechsel einhergehen: Filme, die eigentlich primär für die

53 Im Folgenden wird der englische Begriff für die spezifische historische Praxis beibehalten. Eine Übersetzung ins Deutsche wäre missverständlich - gerade auch mit Blick auf Kolorierungspraktiken in der Stummfilmzeit. 
Kinodistribution produziert worden waren, werden den Marktbedingungen des Fernsehens und des Videomarktes formalästhetisch angepasst. Damit wirft der Vorgang grundsätzlich die Frage auf: „Where does film history really reside?“ (Klawans 1990: 166).

Ganz im Sinne der von Jameson diagnostizierten Stellung von ästhetischen Gütern als Waren werden die nachkolorierten Filme - verstanden als Content beziehungsweise Filminhalt - über diese Praxis aus ihrem historischen Produktionskontext gelöst und einer marktwirtschaftlich orientierten Logik unterstellt - mithilfe von frühen digitalen Technologien der 198oer Jahre und ihren Instrumenten zur Veränderung der Bildästhetik und des Looks.

Auch wenn Veränderung und Anpassung an neue Distributionsbedingungen und -umgebungen in der Filmgeschichte immer schon stattgefunden haben (etwa in der Frühphase des Films in Form von nach regionalen Vorlieben eingefärbten Distributionskopien), so lassen sich dennoch in der Praxis der 1980er Jahre einige wichtige Besonderheiten beobachten. Klawans weist darauf hin, dass hier eine Medientransition - Film zu Videoband - stattfindet. Zudem wird ein computerisierter Bearbeitungsschritt eingeführt. Mit Cesare Brandi gesprochen: Die ästhetische Imago des Films ist bereits vom historischen Träger - dem fotochemischen Filmstreifen - abgelöst und wird in neue mediale Bearbeitungs- und Gebrauchszusammenhänge überführt. ${ }^{54}$

Die neuen institutionellen Rahmenverhältnisse, etwa des Fernsehmarktes, und die technischen Bedingungen (Format, Auflösung) lassen eigene Kriterien der Selektion und der Wertbestimmung von filmischen Objekten entstehen. Dies verbindet sich mit der Annahme der aktualisierenden Institutionen, dass Farbe eine Erweiterung der Faszination des ästhetischen Erlebnisses bedeute. So hat, gemäß Klawans, historisch die Colorization-Technik dadurch ihren Durchbruch erreicht, dass die Bilder eines der wichtigsten ikonischen zeitgeschichtlichen Ereignisse in den 196oer Jahren zum Zwecke des Fundraisings bearbeitet wurden: Die NASA wollte 1970 mit Bildern von der Mondlandung finanzielle Unterstützung einwerben. Da sie fürchtete, dass die Bilder nicht genug faszinative Wirkung entfalten würden, engagierte sie C. Wilson Markle, den Gründer der Firma Image Transform, „to add excitement to the Apollo

54 In diesem Zusammenhang gilt es zu beachten, dass eine Kopie/Übertragung der ästhetischen Imago allein nicht ausreicht, um ein filmisches Kunstwerk als archivarisch gesichert zu verstehen: Insbesondere beim Film ist zu berücksichtigen, dass der (historische) fotochemische Filmstreifen ein wichtiges zu überlieferndes und zu bewahrendes artefaktisches Element des Werkes darstellt. 
mission footage, using his newly invented process of colorization“ (Klawans 1990: $15^{2-153) \cdot 55}$

Der beschriebene Prozess der Colorization wurde 1983 namensgebend für die Firma Colorization Inc mit dem Geschäftsmodell der „Re-Invention“ von Filmen. ${ }^{56}$ So kauften die Gründer Norman und Earl Glick erst den Bestand der Hal Roach Studios, dann im März 1985 einen Bestand von 200 Schwarz-WeißFilmen, die fortan „colorized“ auch auf Videocassetten vertrieben werden sollten. Unter den ersten, wiedererfundenen' Filmen sollten unter anderem die Laurel-und-Hardy-Komödie WAY out West (Horne 1937) und Frank Capras It's A Wonderful Life (1946) sein. Die Tatsache, dass sich ein Regisseur mit Autorenstatus wie Frank Capra auf der Liste fand, setzte die Debatte in Gang, in der die Trademark Colorization zu einem kunstpolitisch höchst umstrittenen Begriff wurde (Klawans 1990: 153). Aber wie schon der Beginn der Praxis bei den Bildern der Mondlandung zeigt, war nicht nur Fiction-Material per Farbe re-aktualisierbar, sondern auch Non-Fiction-Material wurde als ,wieder'erfindbar für den Fernsehmarkt behandelt.

Was der kurze historische Abriss deutlich macht, ist die Problematik der Bewertung des kommerziellen Potenzials ästhetischer Erfahrung, was letztendlich memo- wie kunstpolitische Folgen zeitigt; dies insbesondere in Fragen der Selektion und Kanonisierung. Die Filme wurden in der Logik der ColorizationPraxis vor allem als Entertainment-Commodity gesehen, bei der der Gegenwartswert den Alters- wie Erinnerungswert (vgl. Erläuterungen in $\rightarrow$ Kap 1) überlagert, um das Oxymoron eines „neuen Klassikers“ zu kreieren:

For these interests, colorization is a way to inject the contemporary into the classic, to create the oxymoronic ,new classic', and to sell an old entertainment commodity, for broadcast or video cassette release, with the glittering promise of enhancement through computer technology (Acland 1990: 12).

55 Hier wird deutlich, wie eng die Erfindung der Colorization mit ökonomischen und realpolitischen Interessen verbunden ist, die über eine ästhetische Wirkung und Faszination durchgesetzt werden sollten. Auch tritt in dem Fall hervor, dass die Debatte nicht nur Spielfilme betrifft, sondern auch nichtfiktionales Material, dem noch deutlicher ein Gebrauchswert als ,Dokument' zugesprochen wird, das sich aber gleichzeitig von der faszinativen Bewegtbildwirkung der Colorization nicht trennen lässt.

56 „Their idea, apparently, was to treat old footage, particularly that of television, as a natural resource - to process it like crude oil and market it to distributors“ (Klawans 199o: 153). Vgl. hierzu auch Hediger (2006) mit dem sprechenden Titel The Product that Never Dies. Die Entfristung der kommerziellen Lebensdauer des Films. Insbesondere zu den juristischen, urheberrechtlichen Gründen dieses Wertschöpfungsmodells im Kontext der Praxis der Colorization vgl. Op den Kamp (2015 und 2018). 
Klawans macht in seiner Reflexion über die historische Entwicklung des Konzepts von Kanon deutlich, wie bestimmte Werke über Kopiervorgänge und vor allem auch bestimmte dispositivisch-räumliche Anordnungen eine Wertbestimmung des ,Ausgewähltseins' erfahren. Die Funktionalisierung des Begriffs „Klassiker“ reicht in dieser Perspektive von Fragen der Selektion und Best-Film-Listen, der Prägung des Verständnisses von historischer Dynamik bis hin zur Aufwertung von Film in die Reihe der (antiken oder traditionelleren) Künste (Klawans 199o: 170 ff.; H.-B. Heller 1989). „Klassiker“ als

\begin{abstract}
[t] hat strange, ahistorical category has dominated the debate over colorization from the beginning - from Capra's declaration that his pictures ,are classics because they are superior' to the request by the Directors Guide of Great Britain for governmental protection of a limited number of ,classics', to John Huston's statement that colorization was ,as great an impertinence as for someone to wash flesh tones on a da Vinci drawing.' (The more common comparison was with painting a mustache on the Mona Lisa) (Klawans 1990: 170).
\end{abstract}

„New classic" bedeutet insofern im Rahmen der Colorization-Debatte eine unbestimmte, aber wertsteigernde, nobilitierende Kategorie, in der der jeweilige Film in seiner neuen Umgebung (Videomarkt oder Fernsehprogramm) als alt und neu zugleich vermittelt wird (vgl. hierzu auch die Analyse von Klinger 2006a zum amerikanischen Fernsehprogramm $\rightarrow$ Kap. 3).

Die konkrete Praxis der 198oer Jahre lieferte den Anlass, um die zentralen erinnerungskulturellen und kunstpolitischen Probleme öffentlich zu diskutieren, die für heutige Fragestellungen der digitalen und unter Umständen gar automatisierten Bearbeitungsformen wie Distributions- und Editionsstrukturen wegweisend sind: Wie verändert die neue Zugänglichkeit von historischen Filmen das Verhältnis zur Filmgeschichte und zur Vergangenheit generell? Welche Rolle spielen die Normierungsprozesse (technologisch, ökonomisch, ästhetisch) angesichts des jeweiligen neuen medialen Dispositivs als Bedingung der Sichtbarkeit der Filme?

Auch lassen sich noch heute (wie im Verlauf der späteren Analysekapitel $\rightarrow$ Kap. 4-7 gezeigt werden wird) zentrale Topoi aus der damaligen Diskussion um Vorgehensweisen des Fernsehens über die Colorization-Debatte hinaus feststellen. Hierzu zählen historisch vor allem etwa Formen der Anpassung von Breitwandformaten durch das Pan-und-Scan-Verfahren. So verlor man bei der Praxis des Scannings (aus Sicht der Standards in den 1980ern) einen Teil des Bildes: „In short, they [the technicians, FH] reframe the picture“, um es der Aspect Ratio des TV anzupassen. Panning ist ein ähnliches Verfahren, bei dem vor allem Widescreen-Bilder aufgelöst werden, wenn sich zum Beispiel in Dialogen zwei Schauspieler auf den gegenüberliegenden Seiten des 
ursprünglichen Filmkaders aufhalten und damit außerhalb des im Fernsehen wiedergegebenen Bildes sind: „So, in the process of transferring the film to videotape, a technician pans back and forth from one actor to the other, introducing a camera movement where before there had been a static shot" (Klawans 1990: 161).

Auch in Fragen der Bildauflösung hat das Fernsehen der 1980er technisch eingegriffen. Klawans weist bereits 199 o hoffnungsvoll auf die Möglichkeiten eines zukünftigen HD-Fernsehens in Fragen der Auflösung, aber auch mit Blick auf die Aspect Ratio hin (Klawans 1990: 161). Interessanterweise - das wird auch in der Fallstudie zu DreI NÜSSE FÜr AsChenbröDEL (1973) in $\rightarrow$ Kap. 6 deutlich werden - stellt für historische Filme die Transformation vom lange gebräuchlichen 4:3-Format auf das für digitales Fernsehen normierte 16:9-Format den Dreh- und Angelpunkt einer ähnlichen Debatte um Formattreue in der digitalen Mediendomäne dar.

Klawans interessantes Fazit für die erinnerungskulturellen Folgen einer solchen Filmüberlieferung im Fernsehen lautet: „Even an ideal public-television broadcast of [BATTLEShip, FH] PотEMKIN - without cuts, without commercials, and without color - would be no more than a reminder of the film" (Klawans 199o: 162, Hervorh. FH).

Mit anderen Worten, die Repräsentation eines historischen Films im Fernsehen ist eine (mediale) Form der Erinnerung an den Film im Kino, die durch die Bedingungen der Gegenwart geprägt ist. Zugleich ist aber die Erscheinung im Fernsehen eine (notwendige) Neuaufführung, die unter Umständen neue Generationen von Zuschauern erschließt, sich in deren autobiografische Erinnerung, in das kollektive Gedächtnis und zugleich in die Überlieferungsgeschichte des Werks einschreibt (vgl. hierzu besonders die bereits erwähnte Fallstudie zu DreI NÜSSE FÜr ASCHENBRÖDEL in $\rightarrow$ Kap. 6).

\subsection{Colorized und remastered. Geschichtsbilder im Apparatus TV}

Wenn oben von einer populären Vorstellung von (Film-)Geschichte gesprochen wird, so geschieht dies in Anschluss an Acland (1990), der die Verbindung vom Dispositiv (hier Fernsehen) mit Konzepten des Populären reflektiert. Acland bringt die Praxis der Colorization in Zusammenhang mit der Entwicklung der Populärkultur und ihrem Verhältnis zur Geschichte. ${ }^{57}$ Er macht

57 So untersucht Acland das Verhältnis von „popular cultural history and the notion of tampering, which at once refers to a contempt for cultural heritage and a sense of playfulness, to insolence as well as fascination“ (Acland 1990: 13). 
darauf aufmerksam, dass "technologischer Fortschritt“ willkürlich im Kontext der Colorization-Praxis als ein Alibi benutzt wird, um die „Verbesserung von kulturellen Gebrauchsgegenständen“ zu rechtfertigen. Dies gehe einher mit einer unklaren Vorstellung von Geschichte, die geprägt ist von simplifizierendem Fortschrittsdenken, einem Denken in qualitativen Sprüngen, in dem Maßstäbe der (ökonomischen) Effizienz an ästhetische Wirkung angelegt werden (Acland 1990: 12).

Diese „populären“ Formen im Zeichen des Fernsehens und des Heimvideomarktes grenzt Acland von der Vermittlung von Geschichtskonzepten ab, die sich über die editorische Arbeit von traditionellen Gedächtnisinstitutionen etabliert haben. So beschreibt er Archive und Museen als „eternal resting places", in denen die Artefakte überzeitlich, jenseits momenthafter Interessen existierten (Acland 1990: 14-15).

Als populäre Formen hingegen begreift Acland somit die Produktionsarchive des Fernsehens, die ein anderes Konzept von Geschichte vertreten und vermitteln. In diesem Kontext ist auch die Praxis der Colorization zu sehen. Das Fernsehen und sein Umgang mit Archivmaterial machen damit einen wichtigen Teil der Populärkultur und deren Form von Geschichtsschreibung aus (vgl. hierzu auch noch weitergehend zu Geschichte und Mediendispositiven unter anderem Hickethier 1997 in $\rightarrow$ Kap. 3).

Das Fernsehen bezeichnet Acland in Anlehnung an die englische Übersetzung der Dispositivtheorie zum Kino als Apparatus TV. Dieser Apparatus definiert die institutionellen, ökonomischen, technologischen und ästhetischen Bedingungen als Wiederaufführungs- und Re-Aktualisierungsraum von Filmen. ${ }^{58}$

Mit seiner grundsätzlichen Einschätzung, was die Faszination des Vorgangs ausmacht und warum die Praxis ökonomisch attraktiv erscheint, nimmt Acland eine entscheidende Präzisierung vor, die das Label remastered als Technoimaginäres auch von digitalen Bildern konzeptuell auf den Punkt bringt, da nicht unbedingt der manifeste Qualitätssprung das Entscheidende ist, sondern das Spektakel der (Möglichkeit der) Verbesserung, des Updates: "Contrary to popular opinion, color per se does not attract audiences. It is the colorized - the spectacle of the refinished product, a creation of technological wizardry - that succeeds in doing so" (Acland 1990: 15).

$5^{8}$ "Why are film classics colorized? Two reasons are immediately apparent: (1) because it can be done (technological condition) and (2) because it pays (economic context in which the cost of colorization - approximately $\$ 250$,oo per film and the other determinants of presentation are less than the advertising and video sales possible “ (Acland 1990, 15). 
Es ist das Zusammenwirken von ästhetischen Charakteristika mit binnenästhetischen und paratextuellen Rahmungen, die das historische Objekt zu etwas ,(neu) Gemachtem' (unter den Prämissen der Gegenwart) werden lassen: Der Begriff des ,Erneuerten‘ verbindet sich mit einer Vorstellung von technischem Fortschritt, der sich der menschlichen Fassbarkeit entzieht. Digitale Daten beziehungsweise digitale Bilder leisten dieser Vorstellung aufgrund ihrer Opazität - der fehlenden direkten Sichtbarkeit des zugrunde liegenden Codes (vgl. die vorherigen Abschnitte zu Begriffen und Konzepten in $\rightarrow$ Kap. 2) - Vorschub.

Dies verbindet sich mit Topoi der Technikgeschichte, wie sie H. Böhme (2000) zusammenfasst. Die Referenz auf mythische Figuren und die Vermittlung eines göttlichen, divinen Status sollen - mit Bourdieu gesprochen das symbolische Kapital ( $\rightarrow$ Kap. 7 ) sichern. Das Wunderbare, Traumhafte, Sakrale, Religiöse und Mythische gehören zur Imagologie der Technik. Im Technoimaginären artikulieren sich eschatologische Hoffnungen und Utopien. Hierbei wirken Sehnsüchte nach instrumenteller Rationalität und mythischer Beherrschung der Natur und der Welt paradox zusammen. Zudem kommen Tendenzen der Selbstvergöttlichung zum Ausdruck (H. Böhme 2000: 3-4 FH).

So erfolgt insbesondere bei digitalen Technologien die häufige Referenz auf Magie, Zauberei oder Hexerei. ${ }^{59}$ Bei digitalen Bildern entzieht sich die digitale Qualität der unmittelbaren menschlichen Wahrnehmbarkeit - zumindest mit bloßem Auge - und manifestiert sich dafür in der Performanz des fetischisierenden sinnlichen Spektakels: „the spectacle of refinished“ (Acland 1990: 15$) \cdot 60$

\subsection{Populärkultur und Digitalisierung in der Funktion als Textual Shifter}

Acland (1990) verbindet die Bedeutung der Colorization-Praxis mit Strukturen und Kennzeichen der Populärkultur. Mit Rekurs auf Bennett/Woollacott definiert hierbei Acland die essenzielle Qualität von Populärkultur als eine dynamische; das „,incessantly mobile reordering of the relations' between texts is an essential quality of popular culture: its movement, and, by the same

59 Bezeichnenderweise lautete auch in den 2oooer Jahren ein Werbeslogan des auf Märchen und phantastische Stoffe spezialisierten Disney-Konzerns für seine DVD-Editionen: „Pure Digital Magic“; vgl. Abb. 6.2 in $\rightarrow$ Kap. 6.

6o $\mathrm{Zu}$ der entsprechenden Wahrnehmungsform und -disposition, wie sie mit Blick auf Fetischisierung wirkungsästhetisch modelliert wird, vgl. $\rightarrow$ Kap. 4. 
token, its resistance to the kind of stability signified by museums, archives, catalogues, artists, and originals" (Acland 1990: 16). Vor dem Hintergrund dieser sich ständig reorganisierenden und re-formulierenden soziokulturellen Dynamik wird die Frage nach dem Umgang mit der Versatilität (s. später Distelmeyer 2012, bes. $\rightarrow$ Kap. 5 und $\rightarrow$ Kap. 7) von filmischen Werken - insbesondere unter neuen Medienbedingungen - virulent: Acland zitiert hier das Konzept eines „textual shifter"61 (Acland 1990: 16): Er benennt Colorization als ein Phänomen, das als ein solcher Textual Shifter wirke. Der Begriff dient dazu, (interessegeleitete) Faktoren in ihrer Interaktion und Dynamik zu fassen, die bei der Wiederaufnahme und Neukontextualisierung von präexistenten (historischen) Texten und ästhetischen Phänomenen zusammenwirken: Was wird wie in welcher (medialen) Form von wem wiederaufgenommen?

Aus dieser Perspektive gilt: Colorization sowie aktuelle weitere Praktiken der Transition etwa auf digitale Träger, Wiedergabe- und Speichermedien wirken als Textual Shifter auf die Filme, die (immer wieder) neu aufgegriffen und re-kontextualisiert werden - unter der Maßgabe dynamischer Tendenzen der Populärkultur. Praktiken wie die Colorization und heutige Verfahrensweisen der Filmdigitalisierung sowie deren Folgen sind Beispiele, anhand derer das Zusammengehen von technologischer Entwicklung, medialen Erlebnisformen und soziokulturellen Strukturen deutlich wird; dies gerade deshalb, weil die Phänomene sich an der Schnittstelle zwischen ästhetischer Erfahrung, Gedächtnisformen, abstrakten Geschichtsmodellen und diskursiven und paratextuellen Formationen manifestieren. ${ }^{62}$ Acland schließt seine Überlegungen programmatisch mit dem Blick auf die Möglichkeiten einer Mediengeschichtsschreibung im Zeichen der computergesteuerten Bearbeitung von Bewegtbildern: Die historische Praxis der Colorization sei emblematisch für die Unmöglichkeit einer stabilen Vorstellung von Geschichte(n) (,impossibility of stable histories"). Die Praxis der Colorization nur als eine Banalisierung und Vulgarisierung von Filmen zu sehen, verdeckt die Tatsache, dass das Phänomen - insbesondere auch mit Blick auf die Rolle, den Wert und die

61 Der Begriff Textual Shifter wurde unter anderem zunächst am Beispiel der über Jahrzehnte präsenten und re-formulierten medialen Figur von James Bond in Anschlag gebracht. Das Modell geht ursprünglich zurück auf das linguistische Konzept von Roman Jacobson, in dem, verkürzt dargestellt, eine pragmatische Rückbindung von Bedeutung erfolgt: Die Frage, wer sagt was wann wem. Vgl. Fludernik (1989/99: 97): „The definition provided there identifies as shifters all expressions whose reference can be determined only in relation to the partners of conversation. The referent of I shifts whenever there is a change in the communicative situation, whenever somebody else starts to speak".

62 „Popular memory and popular history should be seen as discursive constructs and contexts, made up for textual shifters“ (Acland 1990: 18). 
Stellung von Filmen - auf das dynamisierende Potenzial von Populärkultur ostentativ hinweist (Acland 1990: 18). Dieses Potenzial spiegelt sich - und dies ist für meine Studie hervorzuheben - in dem Verständnis von Vergnügen und (ästhetischem) Genuss. So stellen Praktiken des digitalen Nachkolorierens implizit einen Aushandlungsort über das jeweilige Verständnis von Modi der Weltwahrnehmung, (ästhetischer) Erfahrung und Erwartungen an sinnliche Unterhaltung dar.

\subsection{Fallbeispiel aus dem Jahr 2012: Die TV-Serie WE LTENBRAND}

Wenn Acland die Verbindung zwischen populärkulturellen Dominanten, ästhetischen Formen, Vorstellungen von (ökonomisch verwertbarer) Unterhaltung und massenmedialer Geschichtsmodellierung hervorhebt, dann lassen sich seine wie auch die weiteren getroffenen Befunde mit einem Beispiel aus der jüngsten Zeit auf gegenwärtige Phänomene applizieren: Der populäre und zugleich sehr umstrittene deutsche Historiker im Dienste des Zweiten Deutschen Fernsehens Guido Knopp verantwortete 2012 unter dem Titel Weltenbrand (2012, TV-Serie, 8 Episoden, ZDF) mit groß angelegter Publicity seine Abschiedsproduktion vor dem Eintritt in seinen Altersruhestand. Die historische Dokumentationsserie schlägt einen narrativen Bogen zwischen den zwei Weltkriegen und benutzt Non-Fiction-Archivbilder, um eigene Thesen zu illustrieren; die ursprünglichen Schwarz-Weiß-Bilder aus dem Archiv wurden - historische Schablonenkolorierungsverfahren imitierend - digital eingefärbt. Zusätzlich wurden die Bilder in der Ästhetik einer Power-Point-Präsentation folienartig ineinander montiert (Abb. 2.1-2.2); dies alles um eine besonders sinnliche und unmittelbare Wirkung zu erzielen: „Emotional rangezoomt" schrieb unter diesen Vorzeichen die Süddeutsche Zeitung (Seibt 2012). ${ }^{63}$

Vor allem die Historiker Bohn/Janz (2012) weisen in ihrer publizistischen Reaktion unter dem Titel „Kann denn Farbe Sünde sein?“ auf das zentrale Spannungsfeld eines solchen Vorgehens hin. Die Spannung entfalte sich zwischen quellenphilologischer Korrektheit und der sinnlich-wirkungsästhetischen

63 „[D]as Verfahren der nachträglichen Kolorierung ist nicht neu. Schon die 2003 in Großbritannien produzierte sechsteilige Dokumentarfilmserie WORLD WAR I IN CoLOR färbte schwarz-weiße Aufnahmen ein. Auch die 2009 ausgestrahlte französische Dokumentarfilmserie Apocalypse über den Zweiten Weltkrieg bediente sich des Verfahrens. Das löste Debatten aus und wurde als Verfälschung historischen Quellenmaterials kritisiert“ (Bohn/Janz 2012). 


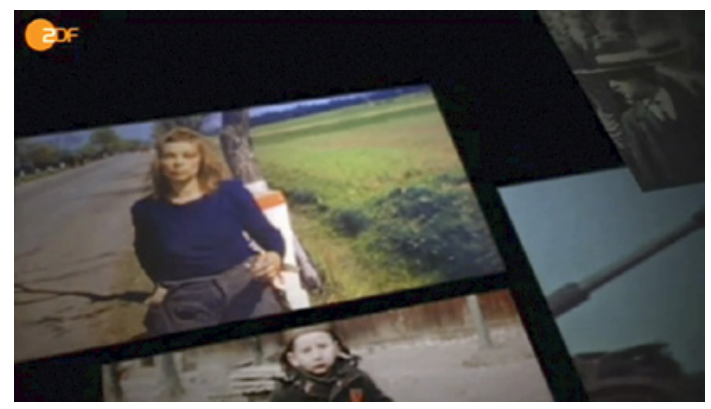

Abb. 2.1

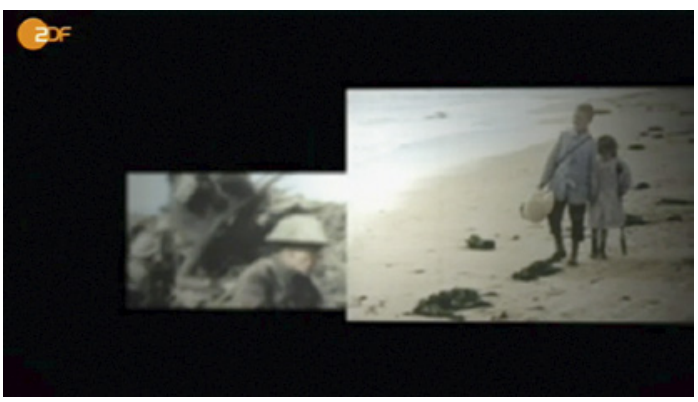

Abb. 2.2

Dimension, der im gegebenen Kontext - quotenorientiertes Fernsehen - eine zentrale diskursformierende Rolle zukommt: „Das ZDF koloriert den Ersten Weltkrieg. Das mindert die Authentizität des Materials - und erhöht die Faszination beim Zuschauer" (Bohn/Janz 2012). So begreifen Bohn/Janz zum einen die nachträgliche Kolorierung als Manipulation von Quellen, was für den Historiker ein Tabu darstellt. Auch "professionelle Hüter des Filmerbes“ wie Restauratoren würden in dem Vorgehen einen Verstoß gegen alle ethischen "Reinheitsgebote" sehen.

Dennoch konstatieren Bohn/Janz zum anderen in der Folge - und verschieben damit die Rahmenbedingungen der Einschätzung der Praxis -, dass die Dokumentarreihe des ZDF keine wissenschaftliche Filmedition sei, sondern „Histotainment“,

das Interesse an Geschichte wecken und ein breites Publikum mit Geschichte informativ unterhalten will. Hier gelten andere Gesetze. Es ist legitim, unter Verwendung historischer Dokumente etwas Neues zu schaffen. Es handelt sich um eine Art Remix (Bohn/Janz 2012).

Was bei Bohn/Janz anklingt, ist eine Modifikation des Referenzrahmens, die auf die Rezeptions- und Erwartungshaltung des Zuschauers angesichts der Bilder zielt. Tatsächlich geht es um Grundsätzlicheres: Unter der Rahmenbedingung massenmedialer Unterhaltung im Fernsehen, zusätzlich 
dispositivisch bedingt durch die Programmierung auf einem Hauptsendeplatz um 20 Uhr 15 und infolge der diskursiven Zuordnung zu einem anderen Genrerahmen („Histotainment"), verschiebt sich der Status der verwendeten (Archiv-)Bilder vom Dokument, das historische Ereignisse ,bezeugt', hin zu der Funktion eines faszinativen Vehikels sinnlicher Erfahrung, das historische Ereignisse im Wahrnehmungseindruck buchstäblich vergegenwärtigt und damit zeitliche Distanz überwindet: „Und so rückt alles sehr nahe, was um Weltalter von uns getrennt schien. Unheimlich“ (Jäger 2012 über Welten BRAND). Das Verhältnis der Gegenwart zu Geschichtlichkeit verändert sich über die mediale Vermittlungsform (Abb. 2.3-2.4).

Abb. 2.3

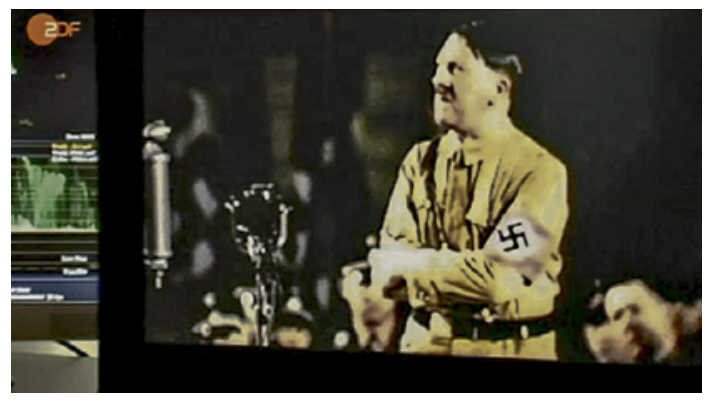

Abb. 2.4

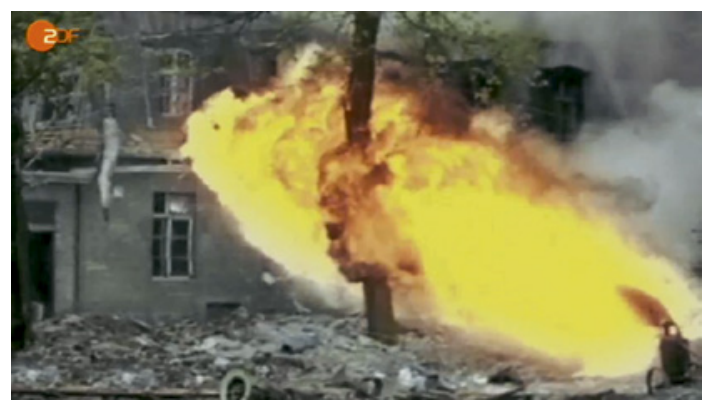

Diese Form der sinnlich-emotionalisierenden audiovisuellen Geschichtsbildmodellierung (vgl. ausführlich zu diesem Begriff in semio-pragmatischer Perspektive $\rightarrow$ Kap. 3 und $\rightarrow$ Kap. 6) ist paratextuell wie publizistisch eng verwoben mit der Praxis der digitalen Aktualisierung, was seinerseits an ein Narrativ von Modernisierung und Fortschritt geknüpft wird:

Die nachträgliche Kolorierung von Schwarz-Weiß-Filmen wird durch die Technologien digitaler Bildbearbeitung ermöglicht. Sie ist Teil eines umfassenderen Phänomens der ,Modernisierung، von Filmen im Zuge der medientechnologischen Entwicklung und hat oft auch das Ziel, neue kommerzielle Verwertungen zu erschließen (Bohn/Janz 2012). 
So sind hier zwei Phänomene festzustellen, die eng aneinandergekoppelt werden: Zum einen manifestieren sich institutionell und ökonomisch imprägnierte - und deshalb unter Bedingungen der Gegenwart generierte ästhetische Formen einer sinnlichen Geschichtsbildvermittlung, zum anderen etabliert sich das Narrativ eines (technologischen) Fortschritts, welcher sich wiederum auf der Zuschauerebene im Modus des Sinnlichen in der Steigerung des Eindrucks medialer Unmittelbarkeit und damit in der phänomenalen Überwindung einer zeitlichen Distanz vermittelt. ${ }^{64}$ Dadurch verweben sich diskursiv und wirkungsästhetisch nicht nur Vorstellungen von Zeit-, Ereignisund Mediengeschichte, auch der Begriff von physisch manifester und erfahrbarer Wirklichkeit wird zum Objekt der Aushandlung. ${ }^{65}$

Wie sehr die argumentativen Ebenen verwoben werden, zeigt die - aus filmtheoretischer Sicht sehr überraschende - Begründung von Guido Knopp für die „technische Verbesserung“ der Quellen. Diese ziele eben auf die ästhetische Wirkung der Bilder und ihre indexikalische Beziehung zur Wirklichkeit:

,Diese Bearbeitung des historischen Materials ist keine Manipulation, sondern eine technische Verbesserung ${ }^{6}$ [...]. Es gehe darum, die Bilder ,aus der Verfälschung der Wirklichkeit, die ja schwarz-weiß bedeutet, in die Wirklichkeit zurück zu holen' (Knopp, zit. n. DAPD in Die Welt 2012).

Damit wird die noch von Acland so genannte "technical wizardry“ der ausgestellten Kolorierung in ein überraschendes Verhältnis zu realistischen Darstellungsformen gesetzt: Die digitale Kolorierung stellt angeblich einen größeren Wirklichkeitsbezug her, da das menschliche Wahrnehmungssystem imitiert wird. Das heißt, wo auf quellenethischer Ebene in das Material eingegriffen wird, soll - wie oben schon betont - auf der ästhetischen Wirkungsebene eine neue Unmittelbarkeit der Wahrnehmung vermittelt werden. Zentral ist aber am Beispiel WeLten BRAND, dass der Stil der Nachkolorierung offensichtlich dem historischen Farbverfahren der Schablonenkolorierung nachempfunden ist. Eine solche Farbgebung ist nicht zwingend an einen mimetischen und indexikalischen Begriff von Realismus gebunden. Damit rekurriert die digitale Kolorierung nicht direkt auf die außermediale menschliche Wahrnehmungsform, sondern auf eine mediengeschichtlich konnotierte.

64 Dies geschieht bei gleichzeitiger Betonung der historischen Distanz auf der diskursiven und sprachlichen Ebene, etwa über die Voice-Over. Zum zeitlich-wechselseitigen Prinzip der Aktualisierung von Archivfilmen Bridging the gap, marking the difference (Kessler 2011) vgl. die Ausführungen in $\rightarrow$ Kap. 5 .

65 Dieser Problemkomplex, der im Verlauf der vorliegenden Studie immer wieder zum Tragen kommen wird, lässt sich weitergehend und vielschichtig mit Paul Ricœurs (2002/2000) Überlegungen zu Geschichtsschreibung und Repräsentation vermitteln. Vgl. hierzu auch $\rightarrow$ Kap. 3 . 
Es findet so eine diskursive und wirkungsästhetische Verflechtung von medienhistorisch konditionierten und konnotierten Wahrnehmungsformen und direkter, außermedialer menschlicher Wahrnehmung statt. Dahinter stehen noch grundsätzlicher Denkformationen, in denen das Verhältnis von menschlicher körperlicher Präsenz, physischen Objekten und Geschichtserleben ausgehandelt wird.

Die Verfahrensweise des zugehörigen paratextuellen Making-of von WeLtenbRAND ist in diesem Kontext aufschlussreich. Über die Kombination aus der dort eingesetzten Voice-Over und der Bildmontage wird das historische Filmmaterial mit der dramatisierend erzählten Geschichte der tatsächlichen Soldatenschicksale verschaltet. Die geschundenen Körper der Kriegsopfer werden in der Narration des Making-of-Films mit dem physisch überlieferten Filmmaterial parallelisiert: Zunächst werden Soldaten an der Front gezeigt, das Filmmaterial - so suggeriert es der dann im Off gesprochene Text - sei auch an der Front gewesen und habe sogar nach seinem langen ,Dienst', Bilder des Krieges zu bewahren, Narben davongetragen.

Das (historische, fotochemische) Filmmaterial wird damit diskursiv als Soldat der Geschichte stilisiert und erfährt eine Anthropomorphisierung. Hier finden vielschichtige Übertragungsprozesse statt: Die Einfühlung in die Kriegsschicksale wird metaphorisch auf das physische Material der Filmstreifen übertragen, was den Film zunächst als Archivfilm, als Filmrolle definiert. Dann wird der Film gescannt und der ,heilenden' Behandlung mit der Software unterzogen. Das fotochemische Material wird in der Folge nur noch symbolisch sichtbar, repräsentiert durch seine bereits digitalisierte Form: Man sieht das gescannte Archivbild umgeben vom Kader eines Tools zur digitalen Bearbeitung (Abb. 2.5-2.7).

Als der ganze Rahmen dann fast vollständig vom Inhalt des Archivbilds eingenommen wird, erfährt das geschundene Bild eine ,Heilung` seiner Narben: Man sieht, wie ein Bearbeiter über digitale Tools einen Kratzer im Bild ausbessert - so werden die Bilder ,verarztet' (Abb. 2.8).

Die ,Heilung der Narben mit digitalen Tools wird im Prozess gezeigt. ${ }^{66}$ Die ,geheilten' Bilder können wieder ihre ganze lebendige Wirkung entfalten, wenn sie bearbeitet in den Montagezusammenhang der Serie eingefügt werden: Film wird so zur Chiffre des ultimativen Zugangs zur Gegenwart des Vergangenen, nicht als mimetisches Dokument, sondern als unmittelbares Erlebnis. ${ }^{67}$

66 Man denke an eine der ursprünglichen Wortbedeutungen von Remediation: ,remediieren` = heilen (siehe zur ursprünglichen Wortbedeutung bei Flückiger 2008 oder Fossati 2009).

67 Die konkreten Vorgänge haben jeweils eine ,remediierende 'Wirkung: Über das Scannen, so die Behauptung des Kommentars, erreiche das Material HD-Qualität, visuell wird aus den einzelnen Rollen eine Montage von verschiedenen Transporträdern, die den Film 

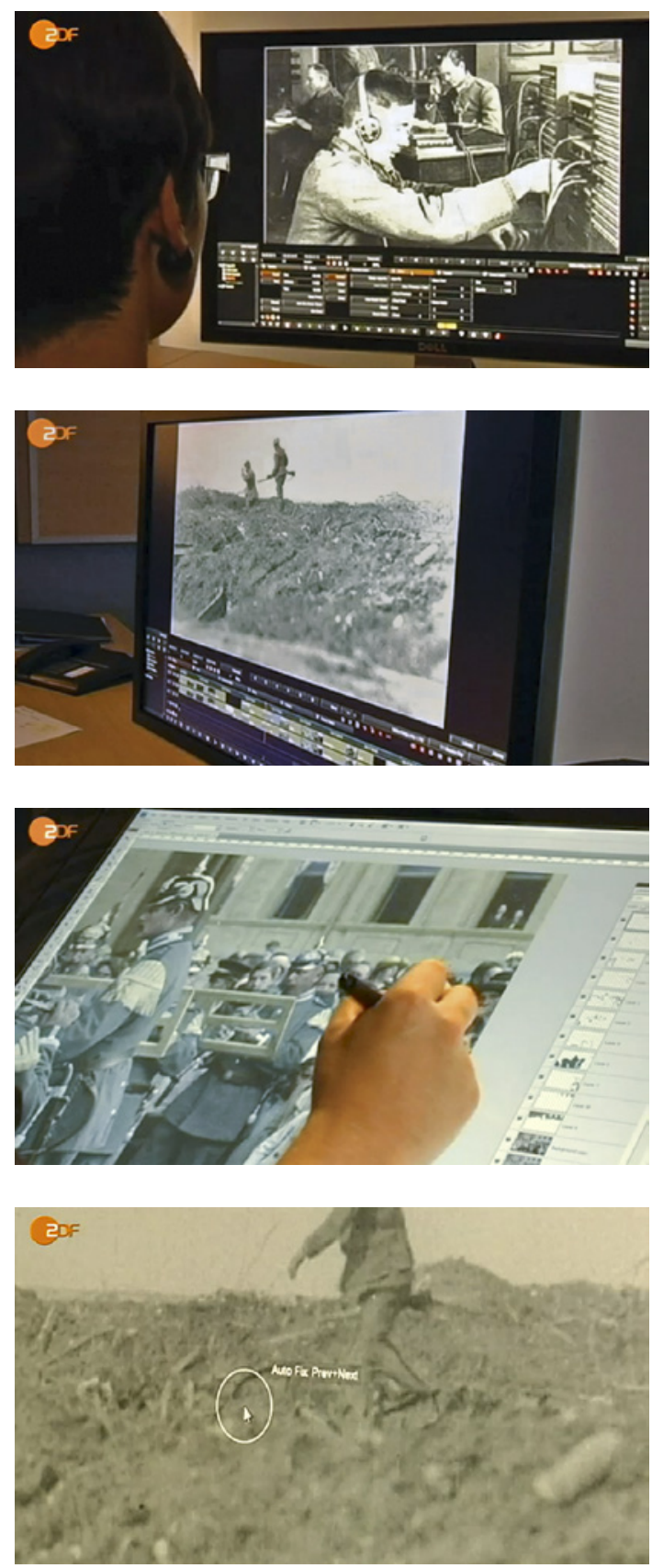

Abb. 2.5

Abb. 2.6

Abb. 2.7

Abb. 2.8 
Die memopolitischen Implikationen dieser Stilisierung des Filmmaterials im Making-of als Überlebender des Krieges lassen sich dahingehend weiter lesen, dass die Reparatur und Wiederbelebung der Bilder implizit eine Heilung der Erinnerung an die Weltkriege bedeutet: Es geht nicht nur darum, vornehmlich die Geschehnisse zu ordnen und zu erklären; vielmehr erhält die emotionale Faszination, dabei gewesen sein zu wollen, ein ganz besonderes Gewicht. Ist unter diesen Vorzeichen dann nicht hier medial vermittelte Geschichte aufgrund der vielfältig aktivierten Ebenen eher als eine Mediengeschichte zu sehen - nicht Geschichte der Medien, sondern über audiovisuelle Medien geschriebene und vor allem sinnlich vermittelte Geschichte?

Diese Mediengeschichte ist als intersubjektive Gedächtnisform zu würdigen - besonders in ihrer Form; gerade weil man sie (massenmedial) über ihre spezifischen Wirkungsdimensionen auch fühlen kann und soll. Und bedeutet dies nicht im konkreten Kontext von WeLtenbrand auch ein memopolitisches Statement: Dass es nun, nach drei Generationen überhaupt erlaubt sei und dies als Wunsch der Zuschauer angenommen wird, sich in das Trauma der beiden Weltkriege einfühlen zu wollen?

Abschließend lässt sich feststellen, dass eine Diskussion um die Grenzziehungen zwischen Unterhaltung und historischem Arbeiten in dem hier vorliegenden Fall den Blick auf die Frage nach der Wirkungsdimension der bearbeiteten Bilder und den darin liegenden ideologischen Implikationen verstellt. Prozesse der Digitalisierung, ihre Vermarktung und Funktionalisierung in einer heutigen Erinnerungskultur gehen eine brisante Verbindung ein, die man insbesondere in der wirkungsästhetischen Dimension als geschichtsmodellierende Form der Memopolitik ernst nehmen sollte (Haber 2011). ${ }^{68}$

So stellt Gustav Seibt in der Süddeutschen Zeitung auch die folgende Frage:

durch den Scanner führen. Die digitale Entfernung von Kratzern wird, wie oben beschrieben, inszeniert. So erfährt das Filmmaterial als ,Überlebender des Krieges` seine Heilung in der (symbolischen) Transition in die digitale Domäne, was in der ,Wiederbelebung durch die Farben kulminiert.

68 Vgl. hierzu auch die Reflexionen von Haber (2011) in Digital Past. Geschichtswissenschaft im digitalen Zeitalter zu dem Verhältnis von Geschichtsforschung und digitalisierten Quellen: „Wo endet die Fachwissenschaft und wo beginnt die sogenannte, interessierte Öffentlichkeit'? Was im analogen Zeitalter sorgsam separierte Bereiche mit verhältnismäßig wenig Kontaktzonen waren, beginnt sich im Zeitalter der Vernetzung zu vermischen [...]. Die zahlreichen Web-Portale, Geschichtsmagazine und TV-Dokumentationen veranschaulichen dies seit Jahren. Entsprechende Ausbildungen im Bereich der Public History beziehen deshalb das neue mediale Umfeld immer mehr in ihre Curricula ein" (Haber 2011:

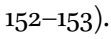


Wer ist der einflussreichste deutsche Historiker der letzten Jahrzehnte? HansUlrich Wehler, Reinhart Koselleck oder Joachim Fest? Keiner der Genannten ist es, wenn man realistisch ist. Es ist Guido Knopp, der Zeitgeschichts- und HistoryDirektor des ZDF, der dort seit dreißig Jahren die Abend- und Nachtprogramme füllt und die Sehgewohnheiten einer ganzen Generation bestimmt hat (Seibt 2012: 1).

Vor diesem Hintergrund entwickelt meine Studie den Ansatz weiter, der bereits von Klawans mit Blick auf die Colorization-Debatte aufgebracht wurde; nämlich die Frage zu stellen, wo und wie sich (Film-)Geschichte entfaltet. Unter Rückgriff auf Acland (1990) ließe sich formulieren, dass die digital kolorierten Bilder von Guido Knopp - ähnlich wie es Bohn/Janz in ihrem Beitrag schreiben - ein Ausdruck ihrer Zeit und der Sehgewohnheiten sind. Wie das Beispiel Weltenbrand gezeigt hat, verweben sich an dieser Stelle massenmediale dispositivische Bedingungen, die wirkungsästhetische Dimension von Bewegtbildern und diskursive, auch paratextuelle Überformungen. Digitale Bildbearbeitung wird zu einem wichtigen Aspekt und Modus der Geschichtsbildmodellierung. Dies ist nicht zuletzt im Zusammenhang mit dem sich steigernden Marktwert dieser Form der Geschichtsbildmodellierung über den Status, etwa digital gefärbt zu sein, zu sehen: Digitale Bildbearbeitungstechnologien erscheinen aus einem solchen Blickwinkel als ultimative Antwort auf die Beweglichkeit, auf sinnliche Bedürfnisse und Sehsüchte populärer Kultur - damit werden aber zugleich vorwaltende Geschichtskonzeptionen in ungeahnte Bewegung versetzt. Was der Beispielkomplex WeltenBRAND (inkl. seiner Paratexte) zusätzlich in seiner Brisanz zum Ausdruck bringt: In dem Cluster aus historischen Filmbildern und digitalen Technologien versteckt sich eine Aushandlung von Vorstellungen physisch manifester, erfahrbarer Realität und der Medialität von Geschichte; dies nachhaltig in der wirkungsästhetischen Dimension, indem der somatisch wirksame Eindruck von Vergegenwärtigung über Bewegtbilder mit dem Technoimaginären von digital verknüpft wird. Was dies für die Konzeption des Zuschauersubjekts bedeutet, wird vor allem Gegenstand der Analysekapitel $\rightarrow$ Kap. 4-7 sein (bes. ausführlich zum Subjektbegriff vgl. $\rightarrow$ Kap. 5). 